\title{
EARLY-TYPE GALAXIES IN THE SLOAN DIGITAL SKY SURVEY. IV. COLORS AND CHEMICAL EVOLUTION
}

\author{
Mariangela Bernardi, ${ }^{1,2}$ Ravi K. Sheth, ${ }^{3,4}$ James Annis, ${ }^{3}$ Scott Burles, ${ }^{3}$ \\ Douglas P. Finkbeiner, $, 6,7$ Robert H. Lupton, ${ }^{6}$ David J. Schlegel, ${ }^{6}$ \\ Mark SubbaRao, ${ }^{1}$ Neta A. Bahcall,${ }^{8}$ John P. Blakeslee, ${ }^{9}$ J. Brinkmann, ${ }^{10}$ \\ Francisco J. Castander, ${ }^{8,11}$ Andrew J. Connolly, ${ }^{4}$ István Csabai, ${ }^{9,12}$ Mamoru Doi, ${ }^{13,14}$ \\ Masataka Fukugita, ${ }^{15,16}$ Joshua Frieman, ${ }^{1,3}$ Timothy Heckman, ${ }^{9}$ \\ Gregory S. Hennessy, ${ }^{17}$ Željko Ivezić, ${ }^{6}$ G. R. Knapp, ${ }^{6}$ Don Q. Lamb, ${ }^{1}$ \\ Timothy McKay, ${ }^{18}$ Jeffrey A. Munn, ${ }^{17}$ Robert Nichol, ${ }^{2}$ Sadanori OKamura, ${ }^{13,19}$ \\ Donald P. SchneIder, ${ }^{20}$ Aniruddha R. ThaKar, ${ }^{10}$ AND Donald G. YorK ${ }^{1}$ \\ Received 2002 September 27; accepted 2002 December 3
}

\begin{abstract}
The colors and chemical abundances of early-type galaxies at redshifts $z<0.3$ are studied using a sample of nearly 9000 galaxies, selected from the Sloan Digital Sky Survey using morphological and spectral criteria. In this sample, redder galaxies have larger velocity dispersions: $g^{*}-r^{*} \propto \sigma^{0.26 \pm 0.02}$. Color also correlates with magnitude, $g^{*}-r^{*} \propto(-0.025 \pm 0.003) M_{r_{*}}$, and size, but these correlations are entirely due to the $L-\sigma$ and $R_{\mathrm{0}}-\sigma$ relations: the primary correlation is color $-\sigma$. The red light in early-type galaxies is, on average, slightly more centrally concentrated than the blue. Because of these color gradients, the strength of the colormagnitude relation depends on whether or not the colors are defined using a fixed metric aperture; the color- $\sigma$ relation is less sensitive to this choice. Chemical evolution and star formation histories of early-type galaxies are investigated using co-added spectra of similar objects. The resulting library of co-added spectra contains spectra that represent a wide range of early-type galaxies. Chemical abundances correlate primarily with velocity dispersion: $\mathrm{H} \beta \propto \sigma^{-0.24 \pm 0.03}, \mathrm{Mg}_{2} \propto \sigma^{0.20 \pm 0.02}, \mathrm{Mg} b \propto \sigma^{0.32 \pm 0.03}$, and $\langle\mathrm{Fe}\rangle \propto \sigma^{0.11 \pm 0.03}$. At fixed $\sigma$, the population at $z \sim 0.2$ had weaker $\mathrm{Mg}_{2}$ and stronger $\mathrm{H} \beta$ absorption compared to the population at $z \sim 0$. It was also bluer. Comparison of these colors and line strengths and their evolution with single-burst stellar population models suggests a formation time of $9 \mathrm{Gyr}$ ago, consistent with a fundamental plane analysis of this sample. Although the fundamental plane shows that galaxies in dense regions are slightly different from galaxies in less dense regions, the co-added spectra and color-magnitude relations show no statistically significant dependence on environment.
\end{abstract}

Key words: galaxies: elliptical and lenticular, cD — galaxies: evolution galaxies: fundamental parameters — galaxies: photometry — galaxies: stellar content

On-line material: color figures, FITS spectra, machine-readable table

\section{INTRODUCTION}

This is the fourth in a series of papers that studies the properties of early-type galaxies at relatively low redshift $z \leq 0.3$. Paper I (Bernardi et al. 2003a) describes how we extracted the sample from the Sloan Digital Sky Survey
(SDSS) database, and how the photometric and spectroscopic parameters (luminosities, effective radii, surface brightnesses, colors, and velocity dispersions) were estimated. It also provides the tables of these parameters. Paper II (Bernardi et al. 2003b) studies the luminosity function and various early-type galaxy correlations in multiple bands

\footnotetext{
${ }^{1}$ Department of Astronomy and Astrophysics, University of Chicago, 5640 South Ellis Avenue, Chicago, IL 60637.

${ }^{2}$ Department of Physics, Carnegie Mellon University, 5000 Forbes Avenue, Pittsburgh, PA 15213.

${ }^{3}$ Fermi National Accelerator Laboratory, P.O. Box 500, Batavia, IL 60510.

${ }^{4}$ Department of Physics and Astronomy, University of Pittsburgh, 3941 O'Hara Street, Pittsburgh, PA 15620.

${ }^{5}$ Department of Astronomy, 601 Campbell Hall, University of California, Berkeley, Berkeley, CA 94720.

${ }^{6}$ Princeton University Observatory, Peyton Hall, Princeton, NJ 08544

${ }^{7}$ Hubble Fellow.

${ }^{8}$ Department of Physics, Yale University, P.O. Box 208101, New Haven, CT 06520.

${ }^{9}$ Department of Physics and Astronomy, Johns Hopkins University, 3400 North Charles Street, Baltimore, MD 21218-2686.

${ }_{10}$ Apache Point Observatory, P.O. Box 59, Sunspot, NM 88349.

${ }^{11}$ Departamento de Astronomía, Universidad de Chile, Casilla 36-D, Santiago, Chile.

12 Department of Physics of Complex Systems, Eötvös Loránd University, Pf. 32, H-1117 Budapest, Hungary.

13 Institute of Astronomy, School of Science, University of Tokyo, Mitaka, Tokyo 181-0015, Japan.

${ }^{14}$ Research Center for the Early Universe, School of Science, University of Tokyo, Tokyo 113-0033, Japan.

${ }^{15}$ Institute for Cosmic Ray Research, University of Tokyo, Kashiwa 277-8582, Japan.

16 Institute for Advanced Study, Einstein Drive, Princeton, NJ 08540.

17 US Naval Observatory, 3450 Massachusetts Avenue, NW, Washington, DC 20392-5420.

18 Department of Physics, University of Michigan, 500 East University, Ann Arbor, MI 48109.

${ }^{19}$ Department of Astronomy, University of Tokyo, Tokyo 113-0033, Japan.

${ }^{20}$ Department of Astronomy and Astrophysics, 525 Davey Laboratory, Pennsylvania State University, University Park, PA 16802.
} 
$\left(g^{*}, r^{*}, i^{*}\right.$, and $\left.z^{*}\right)$. Paper III (Bernardi et al. 2003c) studies the fundamental plane, and its dependence on wavelength, redshift, and environment. In this fourth paper, we study the colors and the spectral line indexes of the galaxies in our sample, both of which correlate strongly with velocity dispersion $\sigma$.

Section 2 presents color-magnitude and color-velocity dispersion relations. It shows that the primary correlation is color- $\sigma$; color-size and color-magnitude relations are a consequence of the fact that size and magnitude correlate with $\sigma$. In $\S 3$, the spectra of the galaxies in our sample are used to study if and how the chemical composition of the early-type galaxy population depends on redshift and environment. The signal-to-noise ratios (S/Ns) of the spectra in this SDSS sample are substantially smaller than the $\mathrm{S} / \mathrm{N}=100$ required to estimate the Lick indices reliably. Therefore, $\S 3.1$ describes the procedure we have adopted for obtaining reliable estimates of absorption line strengths and presents the line indices measurements we used in our analysis. One of the results of this paper is a library of co-added spectra that contains spectra that represent a wide range of earlytype galaxies. This library is available electronically. Sections 3.2 and 3.3 show correlations with velocity dispersion and color, respectively. Section 3.4 compares these measurements with predictions from single burst stellar population models, and $\S 3.5$ studies how these trends depend on environment.

We have chosen to present results for $\mathrm{Mg}_{2}$ (measured in magnitudes) and $\mathrm{Mg} b,\langle\mathrm{Fe}\rangle$, and $\mathrm{H} \beta$ (measured in angstroms), where $\langle\mathrm{Fe}\rangle$ represents an average over Fe5270 and Fe5335. $\mathrm{Mg}_{2}$ and $\mathrm{Mg} b$ are $\alpha$-elements, so, roughly speaking, they reflect the occurrence of Type II supernovae, whereas $\mathrm{Fe}$ is produced in SN Ia. All these line indices depend both on the age and the metallicity of the stellar population (e.g., Worthey 1994), although $\mathrm{Mg}$ and Fe are more closely related to the metallicity, whereas the equivalent width of $\mathrm{H} \beta$ is an indicator of recent star formation. An analysis of other indices on a similar set of co-added SDSS spectra is presented in Eisenstein et al. (2003)

Except where stated otherwise, we write the Hubble constant as $H_{0}=100 \mathrm{~h} \mathrm{~km} \mathrm{~s}^{-1} \mathrm{Mpc}^{-1}$, and we perform our analysis in a cosmological world model with $\left(\Omega_{M}, \Omega_{\Lambda}\right.$, $h)=(0.3,0.7,0.7)$, where $\Omega_{M}$ and $\Omega_{\Lambda}$ are the present-day scaled densities of matter and cosmological constant. In such a model, the age of the universe at the present time is $t_{0}=9.43 h^{-1} \mathrm{Gyr}$.

\section{THE COLOR-MAGNITUDE AND COLOR $-\sigma$ RELATIONS}

The colors of early-type galaxies are observed to correlate with their luminosities, with small scatter around the mean relation (e.g., Baum 1959; de Vaucouleurs 1961; Sandage \& Visvanathan 1978a, 1978b; Bower, Lucey, \& Ellis 1992a, 1992b). In this section, we examine these correlations using the model colors output by the SDSS photometric pipeline. Section 2.1 shows that the color-magnitude relation in our sample is evolving: the population at higher redshift is bluer. It also shows that the primary correlation is actually color with velocity dispersion: color-magnitude and color-size relations arise simply because magnitude and size are also correlated with velocity dispersion. Section 2.2 shows that the color-velocity dispersion relation exhibits no significant dependence on environment. It has been known for some time that giant early-type galaxies are reddest in their cores and become bluer toward their edges (e.g., de Vaucouleurs 1961; Sandage \& Visvanathan 1978a). Therefore, the strength the color-magnitude relation depends on how the color is defined. This is the subject of $\S 2.3$.

\subsection{Galaxy Colors: Evolution}

We begin with a study of the color-magnitude relation in our data set. Estimating the slope of this relation is complicated because our sample is magnitude-limited and spans a relatively wide range of redshifts, and because the slope of the color-magnitude relation is extremely shallow. At any given redshift, we do not have a wide range of magnitudes over which to measure the relation. If we are willing to assume that this relation does not evolve, then the different redshift bins probe different magnitudes, and we can build a composite relation by stacking together the relations measured in any individual redshift bin. However, the shallow slope of the relation means that small changes in color, whether due to measurement errors or evolution, result in large changes in $M$. Thus, if the colors of early-type galaxies evolve even weakly, the slope of the composite color-magnitude relation is drastically affected. We can turn this statement around, of course, and use the color-magnitude relation as a sensitive test of whether or not the colors of the galaxies in our sample have evolved.

Figure 1, the relation between the absolute magnitude in $r^{*}$ and the $g^{*}-r^{*}, r^{*}-i^{*}$, and $r^{*}-z^{*}$ colors, illustrates our argument. We have chosen to present results for these three colors only, since the other colors in our data set are just linear combinations of these, and because, as described in Paper I, the $r^{*}$ band plays a special role in the SDSS

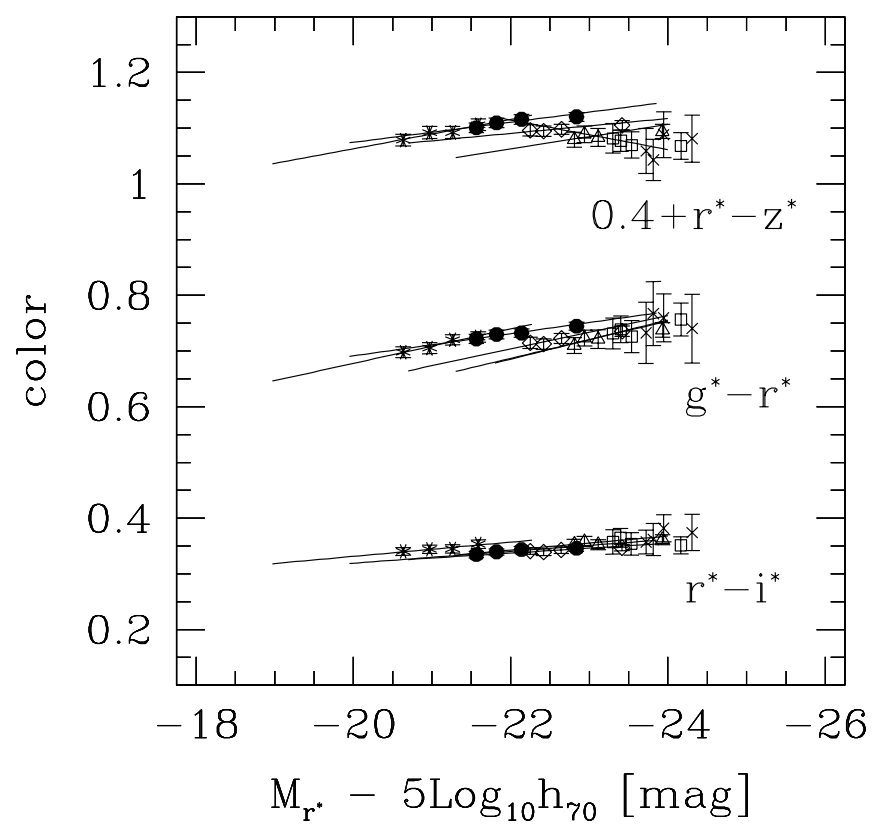

FIG. 1.-Color vs. $r^{*}$ magnitude in volume-limited subsamples. Symbols show the median color at fixed luminosity as measured in the different volume-limited subsamples, error bars show 3 times the uncertainty in this median. Dashed lines show linear fits to the relation in each subsample. The slope of the relation is approximately the same in all the subsamples, although the relations in the more distant subsamples are offset blueward. This offset is greater for the $g^{*}-i^{*}$ colors than for $r^{*}-i^{*}$. Because of this offset, the slope of a line that passes through the relation defined by the whole sample is very different from the slope in each of the subsamples. 
photometry. Briefly, this is the band in which the SDSS spectroscopic sample is selected, and this band has a special status with regard to the SDSS " model" colors (c.f. $\S 2.3$ ).

The figure was constructed by using the same volume-limited subsamples we used when analyzing the $r^{*}$ luminosity function in Paper II. Symbols with error bars show the median, and the error in this median at fixed luminosity in each subsample. Dashed lines show the mean color at fixed magnitude in each subsample; the slopes of these mean relations and the scatter around the mean are approximately the same (we will quantify the slopes of these relations shortly), but the zero points are significantly different. All three color-magnitude relations show qualitatively similar trends, namely a tendency to shift blueward with increasing redshift. For example, $r^{*}-i^{*}$ is bluer by about 0.03 mag in the most distant subsample than in the nearest, whereas the shift in $g^{*}-i^{*}$ is closer to 0.09 mag. Because of the blueward shifts, the slope of a linear fit to the whole catalog, over the entire range in absolute magnitudes shown, is much shallower than the slopes of the individual subsamples.

How much of the evolution in Figure 1 is due to changes in color, and how much to changes in luminosity? To address this, Figure 2 shows the same plot, but with $r^{*}$ magnitude replaced by velocity dispersion. As before, the different dashed lines show fits to the color- $\sigma$ relations in the individual subsamples; the slopes of, and scatter around, the mean relations are similar, but the zero points are different. The magnitude of the shift in color is similar to what we found for the color-magnitude relation, suggesting that the offsets are due primarily to changes in colors rather than luminosity.

At first sight, this might seem surprising, because singleburst models suggest that the evolution in the colors is about one-third that of the luminosities. However, because the slope of the color-magnitude relation is so shallow, even a large change in magnitudes produces only a small shift in the zero point of the colors. To illustrate, let $(C-C *)=$ $-0.02(M-M *)$ denote the color-magnitude relation at the present time. Now let the typical color and magnitude change by setting $C_{*} \rightarrow C_{*}+\delta C$ and $M_{*} \rightarrow M_{*}+\delta M$, but assume that the slope of the color-magnitude relation does not. This corresponds to a shift in the zero point of $0.02 \delta M+\delta C$, demonstrating that $\delta C$ dominates the change in the zero point even if it is a factor of 10 smaller than $\delta M$. (Note that a shallow color-magnitude relation was also obtained with total optical-to-near-infrared colors by Fioc \& Rocca-Volmerange 1999).

To account both for selection effects and evolution, we have computed maximum likelihood estimates of the joint color-magnitude-velocity dispersion distribution, allowing for evolution in the magnitudes and the colors but not in the velocity dispersions: i.e., the magnitudes and colors are

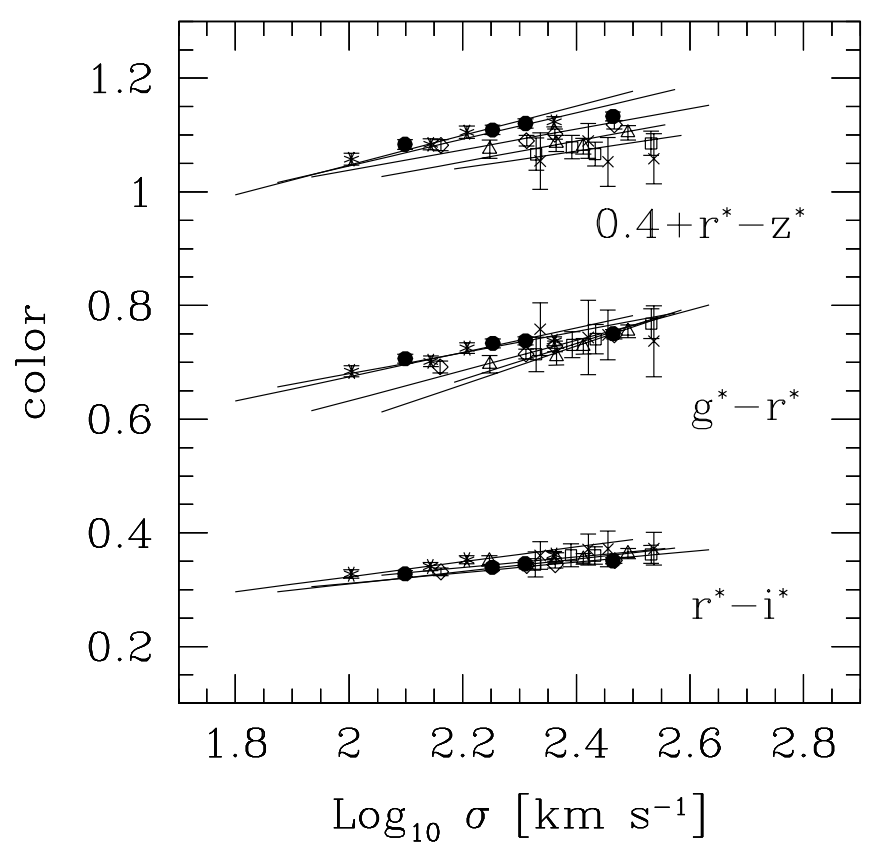

FIG. 2.- Same as Fig. 1, but now showing color vs. velocity dispersion. Redder galaxies have larger velocity dispersions. Dashed lines show that the slope of the relation is approximately the same in all the subsamples, but that the relations in the more distant subsamples are offset blueward. The offset is similar to that in the color-magnitude relations.

assumed to follow Gaussian distributions around mean values that evolve, say $M_{*}(z)=M_{*}-Q z$ and $C_{*}(z)=$ $C_{*}-P z$, but the spread around the mean values, and the correlations between $C$ and $M$ do not evolve. (The maximum likelihood technique we use is described in more detail in Paper II.) We have chosen to only present results for the color $-r^{*}$ magnitude relation, because, as we argue later, color correlates primarily with $\sigma$, which is independent of wave band.

The results are summarized in Table 1. Note that the colors at redshift zero are close to those of the Coleman, Wu, \& Weedman (1980) templates, that the evolution in color is smaller than in magnitude and consistent with the individual estimates of the evolution in the different bands (Table 1 of Paper II shows that $Q=1.15,0.75$, and 0.60 in the $g^{*}, i^{*}$, and $z^{*}$ bands, respectively), and that the best-fit distributions of $M *$ and $V *$ are the same for all three colors and are similar to the values we found in Paper II.

As discussed in Papers II and III, various combinations of the coefficients in Table 1 yield maximum likelihood estimates of the slopes of linear regressions of pairs of variables. Some of these are summarized in Table 2. One interesting combination is the relation between color and magnitude at

TABLE 1

Maximum Likelihood Estimates of the Joint Distribution of Color, $r *$ Magnitude, and Velocity Dispersion AND ITS EVOLUTION

\begin{tabular}{|c|c|c|c|c|c|c|c|c|c|c|c|}
\hline Color & $C_{*}$ & $\sigma_{C}$ & $V_{*}$ & $\sigma_{V}$ & $M *$ & $\sigma_{M}$ & $\rho_{\mathrm{CM}}$ & $\rho_{\mathrm{CV}}$ & $\rho_{\mathrm{VM}}$ & $Q$ & $P$ \\
\hline$g^{*}-r^{*}$ & 0.736 & 0.0570 & 2.200 & 0.1112 & -21.15 & 0.841 & -0.361 & 0.516 & -0.774 & 0.85 & 0.30 \\
\hline$r^{*}-i^{*}$ & 0.346 & 0.0345 & 2.200 & 0.1112 & -21.15 & 0.841 & -0.301 & 0.401 & -0.774 & 0.85 & 0.10 \\
\hline$r^{*}-z^{*}$. & 0.697 & 0.0517 & 2.203 & 0.1114 & -21.15 & 0.861 & -0.200 & 0.346 & -0.774 & 0.85 & 0.15 \\
\hline
\end{tabular}

Notes.-At redshift $z$, the mean values are $C_{*}-P z, M_{*}-Q z$, and $V_{*}$, and the covariances are $\left\langle\left(C-C_{*}\right)\left(M-M_{*}\right)\right\rangle=\sigma_{\mathrm{CM}}^{2}=$ $\sigma_{C} \sigma_{M} \rho_{\mathrm{CM}}$, etc. 
TABLE 2

Maximum Likelihood Estimates of the Slopes and Zero Points of the Color at Fixed Magnitude and Color at Fixed Velocity Dispersion Relations and the Scatter around the Mean Relations

\begin{tabular}{|c|c|c|c|c|c|c|}
\hline \multirow[b]{2}{*}{ COLOR } & \multicolumn{3}{|c|}{ Color- $r *$ Magnitude } & \multicolumn{3}{|c|}{ COLOR-LOG $\sigma$} \\
\hline & Slope & Zero Point & $\mathrm{rms}$ & Slope & Zero Point & $\mathrm{rms}$ \\
\hline$g^{*}-r^{*} \ldots \ldots \ldots \ldots \ldots$ & $-0.025 \pm 0.003$ & 0.218 & 0.053 & $0.26 \pm 0.02$ & 0.154 & 0.0488 \\
\hline$r^{*}-i^{*} \ldots \ldots \ldots \ldots \ldots$ & $-0.012 \pm 0.002$ & 0.085 & 0.033 & $0.12 \pm 0.02$ & 0.072 & 0.0316 \\
\hline$r^{*}-z^{*} \ldots \ldots \ldots \ldots \ldots$ & $-0.012 \pm 0.003$ & 0.443 & 0.051 & $0.16 \pm 0.02$ & 0.343 & 0.0485 \\
\hline
\end{tabular}

fixed velocity dispersion:

$$
\frac{\langle C-\langle C \mid V\rangle \mid M\rangle}{\sigma_{C \mid V}}=\frac{M-\langle M \mid V\rangle}{\sigma_{M \mid V}} \frac{\rho_{\mathrm{CM}}-\rho_{\mathrm{CV}} \rho_{\mathrm{VM}}}{\sqrt{\left(1-\rho_{\mathrm{VM}}^{2}\right)\left(1-\rho_{\mathrm{CV}}^{2}\right)}},
$$

at fixed $V=\log \sigma$. Inserting the values from Table 1 shows that, at fixed velocity dispersion, there is little correlation between color and luminosity. In other words, the colormagnitude relation is almost entirely due to the correlation between color and velocity dispersion.

Figure 3 shows this explicitly. The dashed and dot-dashed lines show fits to the relation between color and magnitude at low (circles) and high (crosses) velocity dispersion (in the plots, the maximum likelihood estimates of the evolution in color and magnitude have been removed). The solid line shows the color-magnitude relation for the full sample, which includes the entire range of $\sigma$; it is considerably steeper than the relation in either of the subsamples. The panel on the right shows the color- $\sigma$ relation at low (circles) and high (crosses) luminosity. The individual fits to the two subsamples are indistinguishable from the fits to the whole sample.

This is also true for the color-size relation, although we have not included a figure showing this. One consequence of this is that residuals from the Faber-Jackson relation corre- late with color, whereas residuals from the luminosity-size relation do not. We will return to this later. Because the primary correlation is color with velocity dispersion, in what follows, we will mainly consider the color- $\sigma$ relation and residuals from it.

While the color- $\sigma$ provides clear evidence that the colors in the high-redshift population in our sample are bluer than in the nearby population, quantifying how much the colors have evolved is more difficult, because the exact amount of evolution depends on the $K$-correction we assume. Appendix A of Paper I discusses how we make our $K$-corrections, as well as what this choice implies for our estimated color evolution.

\subsection{Galaxy Colors: Environment}

Having shown that the colors are evolving and that, to a reasonable approximation, this evolution affects the amplitude but not the slope of the color-magnitude and color- $\sigma$ relations, we now study how the colors depend on environment. To present our results, we assume that environmental effects affect the amplitude more strongly than the slope of the color- $\sigma$ relation. Therefore, we assume that the slope is fixed and fit for the shift in color that best describes the subsample. Figure 4 shows the results. As in Papers I, II, and III, galaxies were divided

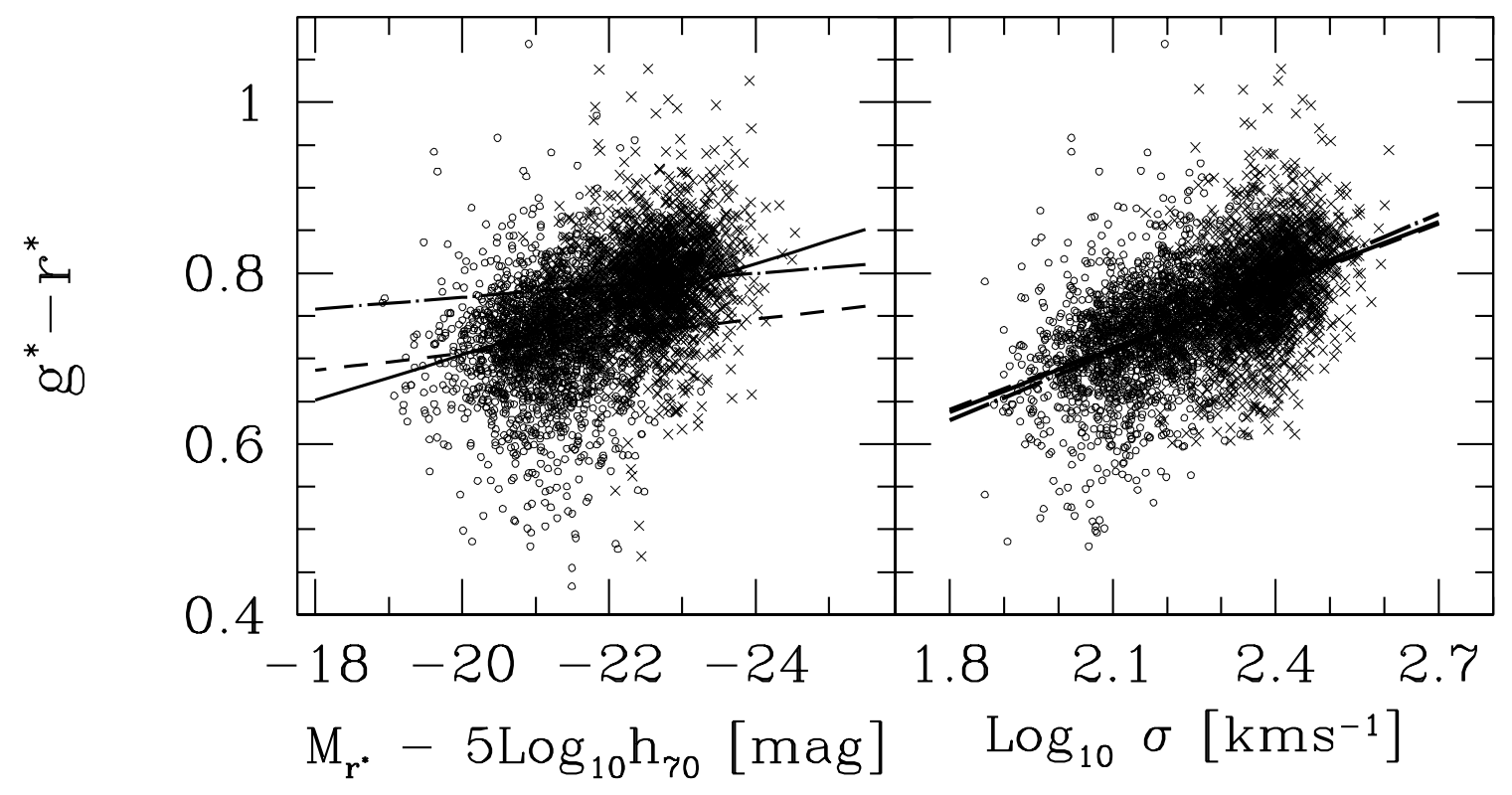

FIG. 3.- Relation between color and magnitude at fixed velocity dispersion (left) and between color and velocity dispersion at fixed magnitude (right). Left: Correlation between color and magnitude is much weaker in the two subsamples than it is for the whole sample, indicating that the color-magnitude relation is driven by the dependence of color and magnitude on velocity dispersion. Right: Individual fits to the two subsamples are indistinguishable from the fits to the whole sample, indicating that the correlation between color and $\sigma$ does not depend on magnitude. 


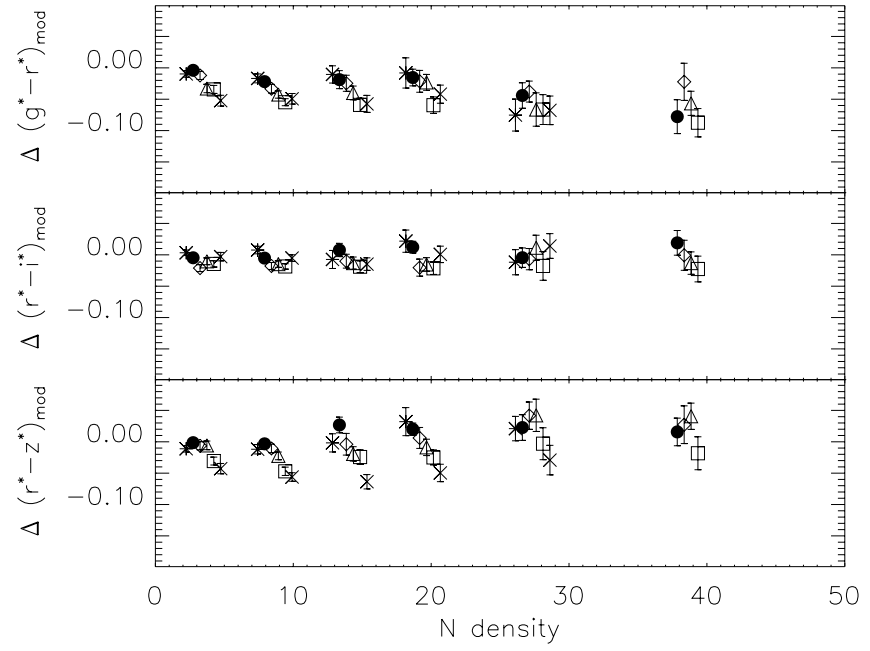

FIG. 4.-Residuals from the color- $\sigma$ relation as a function of local density. At each bin in density, symbols showing results for higher redshifts have been offset slightly to the right. Galaxies at higher redshifts are bluer-hence the trend to slope down and to the right at fixed $N$. The $r^{*}-z^{*}$ colors of galaxies in dense environments are redder than those of their counterparts in less dense regions, although the trend is weaker in the other colors. Although the $g^{*}-r^{*}$ color appears to show the opposite trend, note that the lowest redshift densest bin is the one in which our grouping algorithm is least secure.

into different bins in local density and then further subdivided by redshift (the local density was estimated using the number of near neighbors in coordinate and color space; see Paper I for details). Different symbols in each bin in local density show results for the different redshifts; higher redshifts are offset slightly to the right. This makes trends with evolution easy to separate from those due to environment. In addition to the evolutionary trends we have just discussed, the figure shows that the $r^{*}-z^{*}$ colors are redder in denser regions (bottom), but that this trend is almost completely absent for the other colors. The $g^{*}-r^{*}$ color appears to be slightly bluer in the densest region lowest redshift bin, where our grouping procedure is least secure.

The tightness of the color-magnitude relation of cluster early types has been used to put constraints on the ages of cluster early types (e.g., Kodama, Bower, \& Bell 1999). Figure 5 shows how the thickness of this relation depends on environment. The plot shows no evidence that the scatter around the mean relation decreases slightly with increasing density; a larger sample is needed to make conclusive quantitative statements about this and about whether or not the scatter around the mean relation depends more strongly on environment at low than at high redshift.

\subsection{Color Gradients and the Color-Magnitude Relation}

It has been known for some time that giant early-type galaxies are reddest in their cores and become bluer toward their edges (e.g., de Vaucouleurs 1961; Sandage \& Visvanathan 1978a). Figure 3 of Paper I shows that the half-light angular sizes of the galaxies in our sample are indeed larger in the bluer bands. Figure 6 shows how the effective physical radii of the galaxies in our sample change in the four bands. On average, early-type galaxies have larger effective radii in the bluer bands. This trend indicates that there are color gradients in early-type galaxies. The

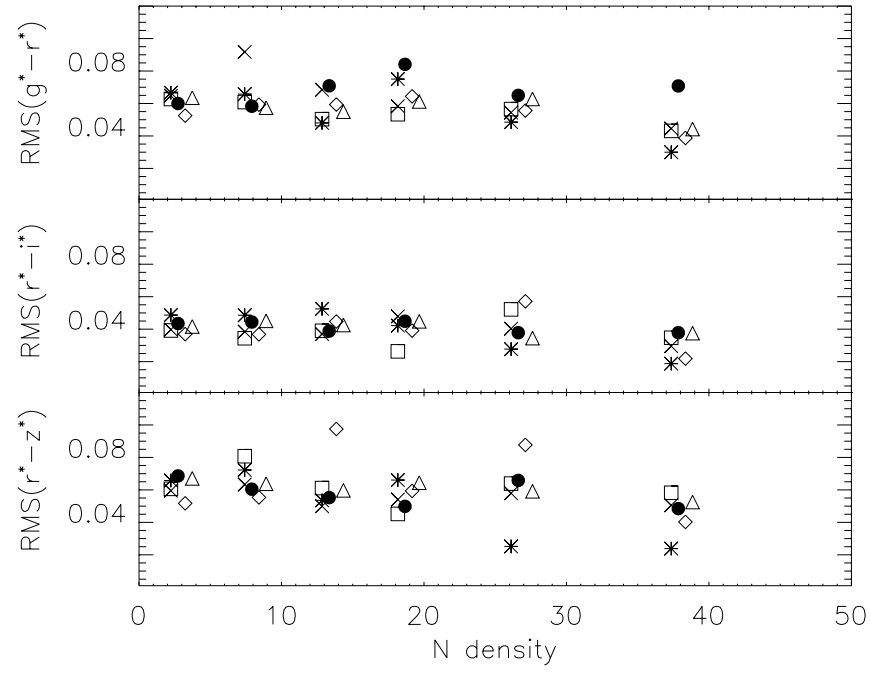

FIG. 5.-Same as for Fig. 4, but now showing the thickness of the color- $\sigma$ relation as a function of local density.

distribution of size ratios does not correlate with luminosity. However, the ratio of the effective size in the $g^{*}$ and $r^{*}$ bands is slightly larger for bluer galaxies than for redder ones, suggesting that color gradients are stronger in the galaxies that are bluer. In addition, the scatter around the mean ratio is slightly larger for the bluer galaxies.

As Scodeggio (2001) emphasizes, if the effective sizes of galaxies depend on wave band, then the strength of the color-magnitude relation depends on how the color is defined. Therefore, we have tried five different definitions for the color. The first uses the total luminosities one infers from fitting a de Vaucouleurs model to the light in a given band. The "total" colors defined in this way are relatively noisy, because they depend on independent fits to the sur-

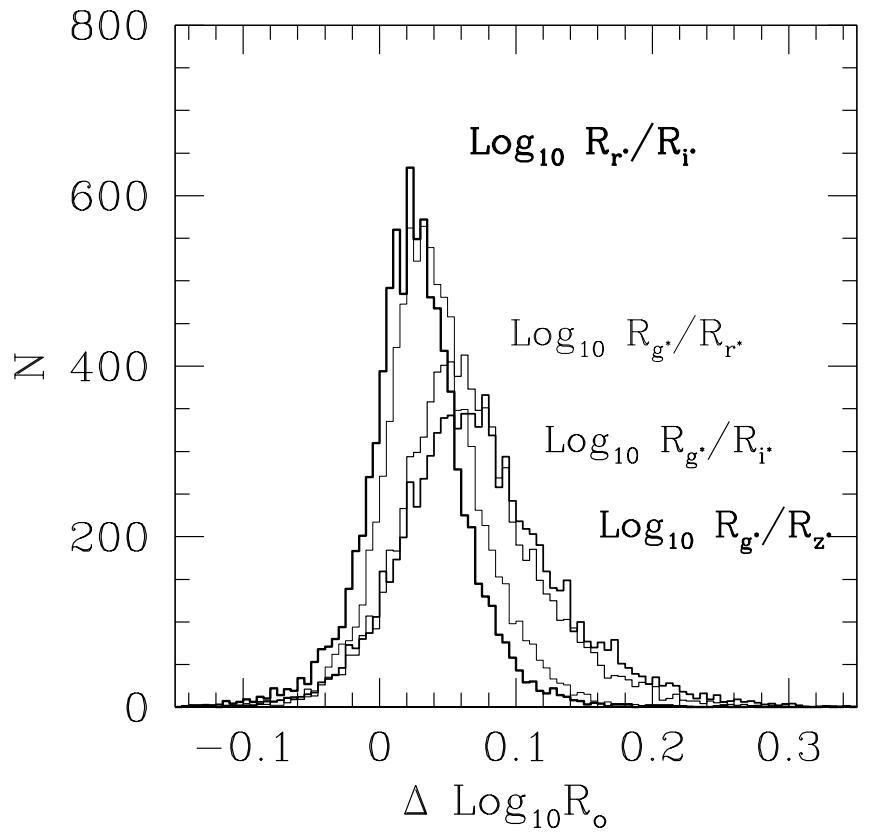

FIG. 6.-Differences between the effective sizes of galaxies in different bands; the blue light is less centrally concentrated than the red light. 
face brightness distributions in each band (c.f. discussion in Paper I). Since the half-light radius is larger in the bluer bands, a greater fraction of the light in the redder bands comes from regions that are closer to the center than for the bluer bands. Therefore, this total color can be quite different from that which one obtains with a fixed angular or physical aperture.

To approximate fixed physical aperture colors, we have integrated the de Vaucouleurs profiles in the different bands assuming a top-hat filter (since this can be done analytically) of scale $f$ times the effective $r^{*}$ radius, $R_{o}\left(r^{*}\right)$, for a few choices of $f$. The resulting colors depend on $f$, and the slope of the associated color magnitude relation decreases as $f$ decreases. We have arbitrarily chosen to present results for $f=2$. These are not quite fixed aperture colors, since the effective angular aperture size varies from one galaxy to another, but, for any given galaxy, the aperture size is the same in all the bands (i.e., it is related to the effective radius in $\left.r^{*}\right)$.

A third color is obtained by using the light within a fixed angular aperture, which is the same for all galaxies. The "fiber" magnitudes output by the SDSS photometric pipeline give the integrated light within a 3 " aperture, and we use these to define the "fiber" color. A fourth color is that computed from the Petrosian magnitudes output by the SDSS photo pipeline (Stoughton et al. 2002).

A fifth color uses the "model" magnitudes output by the SDSS photometric pipeline. These are close to what one might call fixed aperture colors, because they are obtained by finding that filter which, given the $\mathrm{S} / \mathrm{N}$, optimally detects the light in the $r^{*}$ band, and then using that same filter to measure the light in the other bands (which is one reason why they are less noisy than the total color defined above). (By definition, the model and total de Vaucouleurs magnitudes are the same in $r^{*}$. They are different in other bands because the effective radius is a function of wavelength. We have verified that the difference between these two magnitudes in a given band correlates with the difference between the effective radius in $r^{*}$ and the band in question.) In this respect, the model colors are similar to those one might get with a fixed physical aperture (they would be just like the fixed physical aperture colors, if the optimal smoothing filter was a top hat). These, also, are not fixed angular aperture colors, since the effective aperture size varies from one galaxy to another, but, for any given galaxy, the aperture is the same in all the bands.

A final possibility is to use "spectral magnitudes"; these can be made by integrating up the light in the spectrum of each galaxy, weighting by the different passband filters. Whereas the other five colors require a good understanding of the systematics of the photometric data sets, this one requires a similar understanding of the spectroscopic data sets also. We have not done this here, although this should be possible in the near future.

The resulting $g^{*}-r^{*}$ color-magnitude relations are shown in Figure 7. The $x$-axis in the top two panels is the de Vaucouleurs' magnitude in $r^{*}$, whereas it is the fiber magnitude in $r^{*}$ in the third panel, the Petrosian $r^{*}$ magnitude in the fourth panel, and the model $r^{*}$ magnitude in the bottom panel. So that evolution effects do not combine with the magnitude limit of our sample to produce a shallow relation, we divided our sample into two: a low-redshift sample, which includes all galaxies at $z \leq 0.08$, and a high-redshift

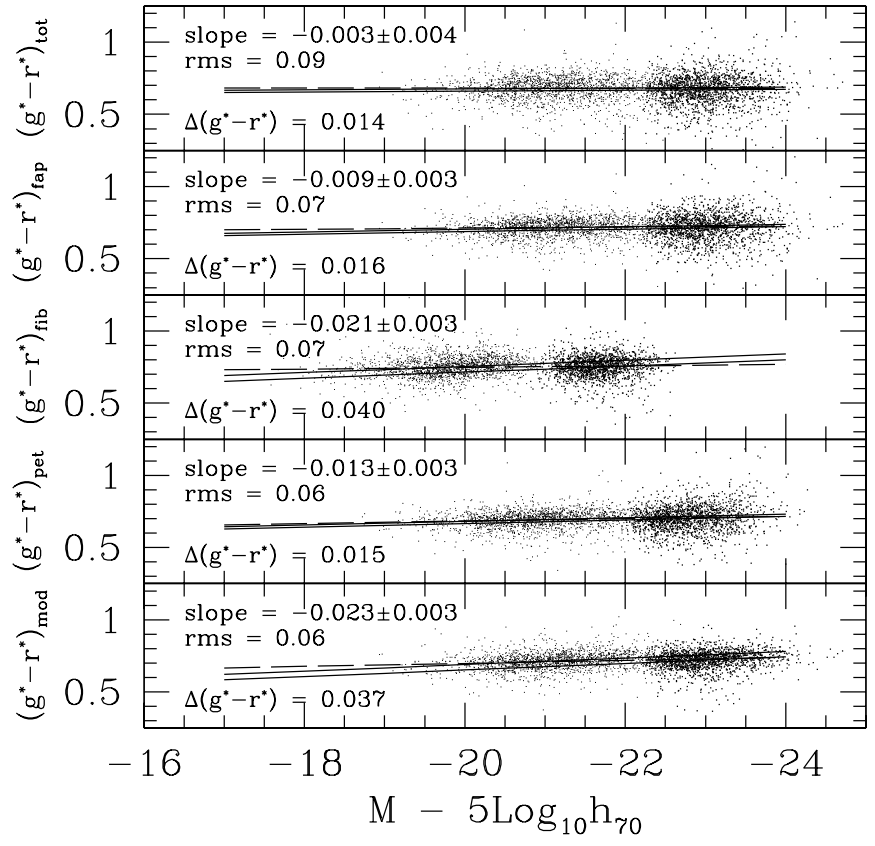

FIG. 7.-Color-magnitude relations associated with various definitions of magnitude and $g^{*}-r^{*}$ color. Top left of each panel shows the slope determined from a low-redshift subsample. Fixed-aperture colors (bottom) give steeper color-magnitude relations; the correlation is almost completely absent if colors are defined using the total magnitudes (top). Bottom left of each panel shows the zero-point shift required to fit the higher redshift sample. This shift is an estimate of how the colors have evolved-it, too, depends on how the color was defined. Dashed lines show fits to the whole sample; because they ignore the evolution of the colors, they are significantly shallower than fits that are restricted to a small range in redshifts, for which neglecting evolution is a better approximation.

$z \geq 0.16$ sample. For each definition of color, we computed the slope and amplitude of the color-magnitude relation in the low-redshift sample. This slope is shown in the top left corner of each panel. We then required the slope of the high-redshift sample to be the same (recall from Fig. 1 that this is a good approximation); the offset required to get a good fit is shown in the bottom left of each panel. This is the quantity that provides an estimate of how much the colors have evolved. The two thin solid lines in each panel show the low- and high-redshift color-magnitude relations computed in this way. For comparison, the dashed line shows a fit to the full sample, ignoring evolution effects; in all the panels, it is obviously much flatter than the relation at low redshift.

The figure shows clearly that the slope of the color-magnitude relation depends on how the color was defined: it is present when fixed apertures are used (e.g., bottom), and it is almost completely absent when the total light within the de Vaucouleurs' fit is used (top). Our results are consistent with those reported by Okamura et al. (1998) and Scodeggio (2001). Note that one's inference of how much the colors have evolved, $\Delta\left(g^{*}-r^{*}\right)$, also depends on how the color was defined.

A similar comparison for the correlation between color and velocity dispersion $\sigma$ is presented in Figure 8. We have already argued that color- $\sigma$ is the primary correlation; this relation is also considerably less sensitive to the different definitions of color. However, it is sensitive to evolution: a fit to the full sample gives a slope of 0.14 , compared with the value of 0.23 for the low-redshift sample. Because the mean 


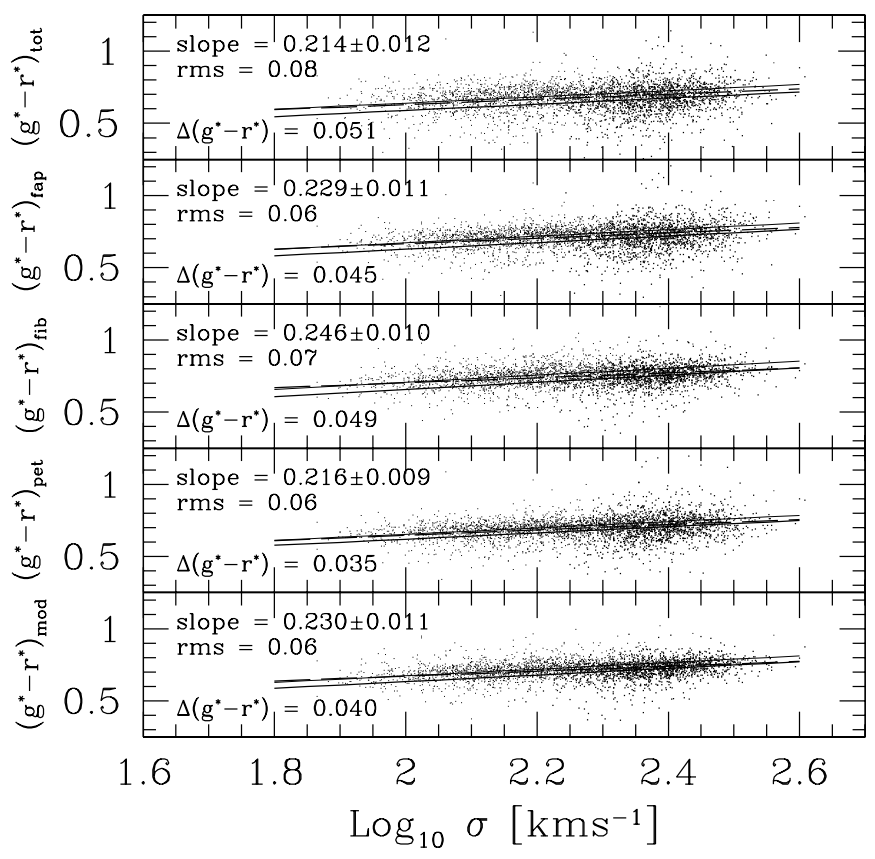

FIG. 8.- Same as for Fig. 7, but now showing how the color- $\sigma$ relation varies as the definition of $g^{*}-r^{*}$ color changes.

color- $\sigma$ relation is steeper than that between color and magnitude, the change to the slope of the relation is less dramatic. The zero-point shifts, which estimate the evolution of the color, are comparable both for the colormagnitude and the color $\sigma$ relations, provided the SDSS model colors are used (bottom).

\section{LINE INDICES: CHEMICAL EVOLUTION AND ENVIRONMENT}

We now turn to a more detailed study of the spectra in our sample. To measure spectral features reliably requires a spectrum with a higher $\mathrm{S} / \mathrm{N}$ than we have for any individual galaxy in our sample. Section 3.1 describes the procedure we have adopted to deal with this. All the line indices we study below correlate with velocity dispersion $\sigma$. Because $\sigma$ correlates with luminosity, the magnitude limit of our sample means that we have no objects with low-velocity dispersions at high redshifts. By presenting results at fixed velocity dispersion, our analysis of line indices should not be biased by this selection: this is the subject of $\S 3.2$.

\subsection{Composite Spectra of Similar Objects}

The typical $\mathrm{S} / \mathrm{N}$ of the spectra in our sample is about 15 (Fig. 18 in Paper I). To measure spectral features reliably requires a spectrum with $\mathrm{S} / \mathrm{N} \sim 100$ (e.g., Trager et al. 1998), so we have adopted the following procedure.

We have a large number of galaxies in our sample, many of which have similar luminosities, sizes, velocity dispersions, and redshifts. By co-adding the spectra of similar galaxies, we can produce a composite spectrum with a considerably higher $\mathrm{S} / \mathrm{N}$. Since we wish to increase the $\mathrm{S} / \mathrm{N}$ by a factor of about 7, we need at least 50 galaxies per composite spectrum. On the other hand, we do not want to coadd spectra of galaxies that differ considerably from each other. Therefore, we divided the galaxies in our sample into five bins each of redshift, luminosity, velocity dispersion, effective radius, and density, and co-added the spectra of all the galaxies in each bin. This gave about 200 composite spectra, with varying numbers of galaxies contributing to each. We then excluded from further consideration all composites that had $\mathrm{S} / \mathrm{N}<50$. Figure 9 shows a selection of the 182 composite spectra with $\mathrm{S} / \mathrm{N}>50$ (for given bins in $\sigma$ and $z$, we show the composite spectrum that has the median $\mathrm{S} / \mathrm{N}$ ). The line at the bottom of each panel shows the rms scatter of the individual spectra used to make the composite spectrum. These 182 composite spectra, the scatter, and the errors, as a function of rest-frame wavelength, are available in the electronic edition of the Journal.

We estimated the $\mathrm{Mg}_{2}, \mathrm{Mg} b, \mathrm{H} \beta$, and $\langle\mathrm{Fe}\rangle$ line indices in the higher $\mathrm{S} / \mathrm{N}$ composite spectra following methods outlined by Trager et al. (1998). (Analysis of the properties of early-type galaxies using these higher $\mathrm{S} / \mathrm{N}$ composite spectra is ongoing.) The estimated indices were aperturecorrected following Jørgensen (1997): $\mathrm{Mg}_{2}=\mathrm{Mg}_{2}^{\text {est }}$ $+0.04 \log _{10}\left[1.5 /\left(r_{o} / 8\right)\right], \mathrm{H}_{\beta}=\mathrm{H}_{\beta}^{\mathrm{est}}\left[1.5 /\left(r_{o} / 8\right)\right]^{-0.005},\langle\mathrm{Fe}\rangle=$ $\langle\mathrm{Fe}\rangle^{\text {est }}\left[1.5 /\left(r_{o} / 8\right)\right]^{0.05}, \quad$ and $\langle\operatorname{Mg} b\rangle=\langle\mathrm{Mg} b\rangle^{\text {est }}[1.5 /$ $\left.\left(r_{o} / 8\right)\right]^{0.05}$. (Because the indices were measured for co-added spectra, we use the mean values of $r_{o}$ in each bin to make the aperture correction.) In addition, the observed line indices of an individual galaxy are broadened by the velocity dispersion of the galaxy. Simulations similar to those we used to estimate the velocity dispersion itself (see Appendix B in Paper I) were used to estimate and correct for the effect of the broadening. For all the indices presented here, the required corrections increase with increasing $\sigma$. (We use the mean value of $\sigma$ in each bin to make the corrections.) Whereas the corrections to $\mathrm{Mg}_{2}$ and $\mathrm{H} \beta$ are small (on the order of a percent), the corrections to $\mathrm{Mg} b$ and $\mathrm{Fe}$ are larger (on the order of $10 \%$ ).

Table 3 summarizes our line index measurements. Column (1) gives the ID number of the composite spectrum; columns (2)-(6) give the centers of the bins in velocity dispersion, size, absolute magnitude, redshift, and local density that were used to define which galaxies contribute to the composite; and columns (7)-(14) give the measured strength of the index and the associated error on the measurement, for $\mathrm{H} \beta, \mathrm{Mg}_{2}, \mathrm{Mg} b$, and $\langle\mathrm{Fe}\rangle$.

\subsection{Correlations with Velocity Dispersion}

Figures 10 and 11 show how the line indices in Table 3 correlate with velocity dispersion. In all panels, stars, filled circles, diamonds, triangles, squares, and crosses show the redshift bins $z<0.075,0.075<z \leq 0.1,0.1<$ $z \leq 0.12,0.12<z \leq 0.14,0.14<z \leq 0.18$, and $z>0.18$. The median redshifts in these bins are 0.062, 0.086, $0.110,0.130,0.156$, and 0.200 . For clarity, at each bin in velocity dispersion, the symbols for successive redshift bins have been offset slightly to the right from each other. This should help to separate out the effects of evolution from those that are due to the correlation with $\sigma$. The solid line and text in each panel shows the relation that is obtained by performing simple linear fits at each redshift and then averaging the slopes, zero points, and rms scatter around the fit at each redshift. The text at top right of each panel shows the shift between the lowest and highest redshift bins, averaged over the values at $\log \sigma=2.2, \quad 2.3$, and 2.4. Roughly speaking, this means that the shifts occur over a range of about 

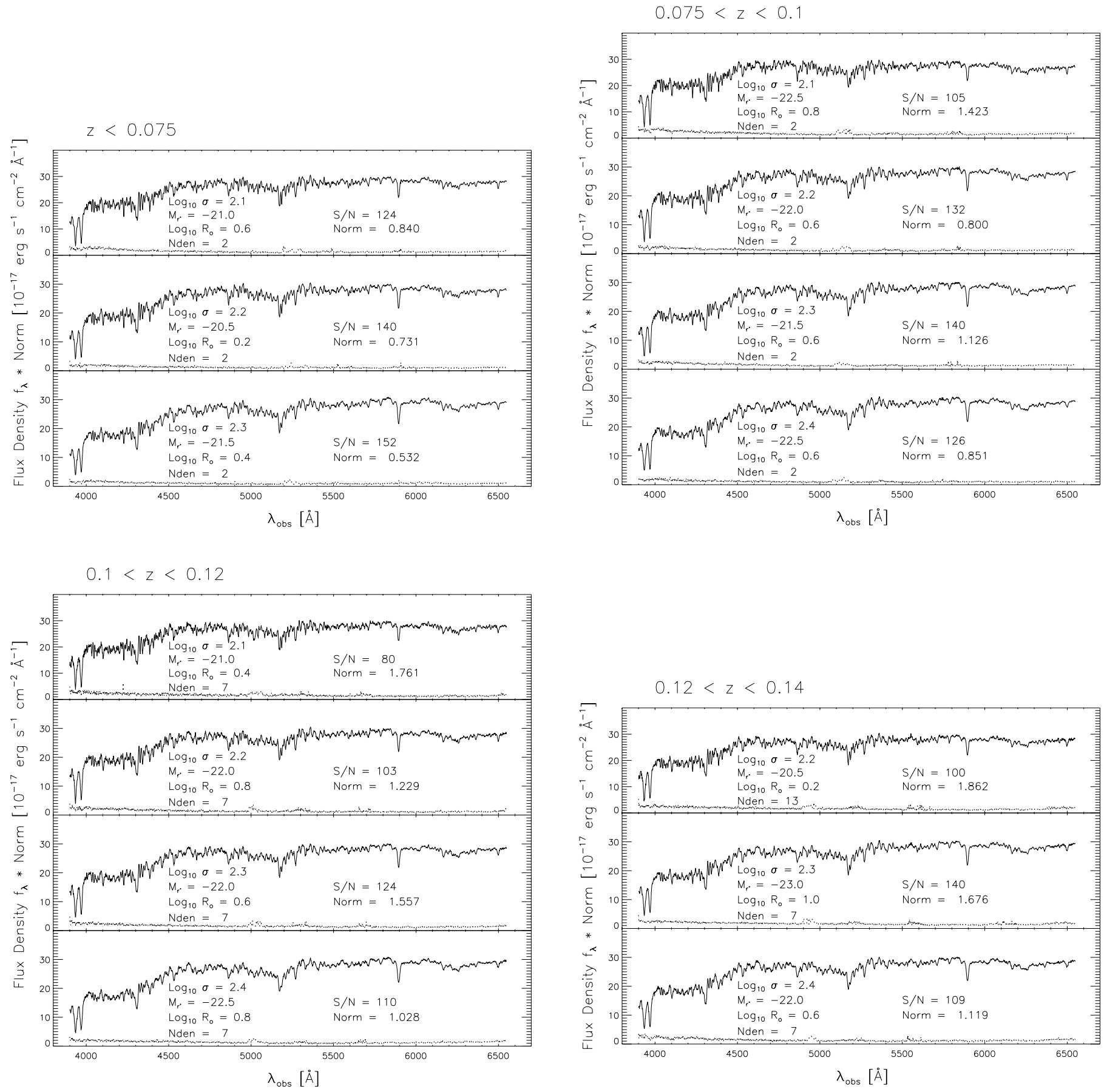

FIG. 9.-Composite spectrum obtained by co-adding the spectra of galaxies with similar redshifts, velocity dispersions, absolute magnitudes, effective radii, and local densities. The line at the bottom of each panel shows the rms scatter of the individual spectra used to make the composite spectrum. The $\mathrm{S} / \mathrm{N}$ of the composite spectrum is also shown.

$0.2-0.06=0.14$ in redshift, which corresponds to a time interval of $1.63 \mathrm{Gyr}$.

Figure 10 shows the well-known correlation between $\mathrm{Mg}_{2}$ and $\sigma$ : at fixed redshift, $\operatorname{Mg}_{2} \propto \sigma^{0.20 \pm 0.02}$ with a scatter around the mean relation at each redshift of $0.011 \mathrm{mag}$. The fit we find is similar to that found in previous work based on spectra of individual (as opposed to co-added) galaxies (e.g., Jørgensen 1997; Bernardi et al. 1998; Pahre et al. 1998; Kuntschner 2000; Blakeslee et al. 2001; Bernardi et al. 2002), although the scatter we find is somewhat smaller. The slope of our fit is shallower than that reported by Colless et al. (1999), but this may be the consequence of our decision to perform linear regression, rather than maximum likelihood, fits. (Maximum likelihood fits are difficult at the present time because our bins in luminosity are rather large. We plan to make the maximum likelihood estimate when the sample is larger, so that finer bins in luminosity can be made.)

Although the magnitude limit of our sample makes it difficult to study the evolution of the $\mathrm{Mg}_{2}-\sigma$ relation, a few bins in $\sigma$ do have galaxies from a range of different redshifts. Recall that, for the purposes of presentation, the points in 
$0.14<z<0.18$
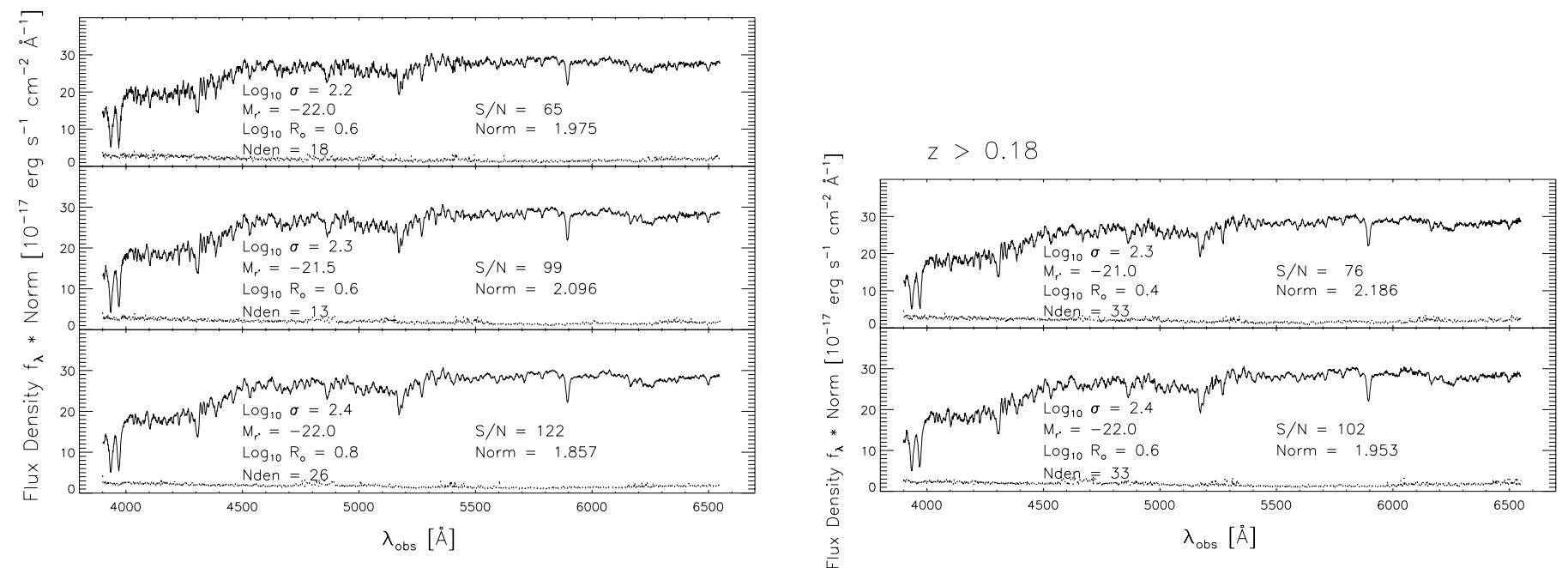

FIG. 9.-Continued

each bin in $\sigma$ have been shifted to the right by an amount that depends on the redshift bin they represent. When plotted in this way, the fact that the points associated with each bin in $\sigma$ slope down and to the right suggests that, at fixed $\sigma$, the higher redshift galaxies have smaller values of $\mathrm{Mg}_{2}$. Large values of $\mathrm{Mg}_{2}$ are expected to indicate either that the stellar population is metal-rich, or old, or both. Thus, in a passively evolving population, the relation should be weaker at high redshift. This is consistent with the trend we see. The average value of $\mathrm{Mg}_{2}$ decreases by about $0.015 \pm 0.004$ mag between our lowest and highest redshift bins (a range of about $1.63 h^{-1} \mathrm{Gyr}$ ). We will return to this shortly.

The top panel of Figure 11 shows that, at fixed redshift, $\mathrm{Mg} b \propto \sigma^{0.32 \pm 0.03}$, with a scatter of 0.020 . This is consistent with the scaling reported by Trager et al. (1998). [A plot of $\mathrm{Mg} b$ vs. $\mathrm{Mg}_{2}$ is well fit by $\log \mathrm{Mg} b=$ $(1.41 \pm 0.18) \mathrm{Mg}_{2}+0.26$; this slope is close to the value $0.32 / 0.20$ one estimates from the individual $\mathrm{Mg} b-\sigma$ and $\mathrm{Mg}_{2}-\sigma$ relations. It is also consistent with Fig. 58 in Worthey (1994).] As was the case for $\mathrm{Mg}_{2}$, our data indicate that, at fixed velocity dispersion, $\mathrm{Mg} b$ is weaker in the higher redshift population. The average difference between our lowest and highest redshift bins is $0.030 \pm 0.012$. This corresponds to a fractional change in $\mathrm{Mg} b$ of 0.07 over about $1.63 h^{-1}$ Gyr. In contrast, Bender, Ziegler, \& Bruzual (1996) find that $\mathrm{Mg} b$ at $z=0.37$ is smaller by $0.3 \AA$ compared with the value at $z=0$. This is a fractional change of about 0.07 but over a redshift range that corresponds to a time interval of $4 h^{-1}$ Gyr. Bender et al. also reported weak evidence of differential evolution: the low- $\sigma$ population appeared to have evolved more rapidly. Our $\mathrm{Mg}_{2}-\sigma$ and $\mathrm{Mg} b-\sigma$ relations also show some evidence of such a trend.

Colless et al. (1999) define $\mathrm{Mg} b^{\prime}=-2.5 \log (1-\mathrm{Mg} b /$ 32.5) and show that their data are well fitted by $\mathrm{Mg}$ $b^{\prime} \propto(0.131 \pm 0.017) \log \sigma-(0.131 \pm 0.041)$ with a scatter around the mean relation of 0.022 mag. Kuntschner (2000) shows that the galaxies in the Fornax Cluster follow this same scaling, although the scatter he finds is $0.011 \mathrm{mag}$. Our co-added spectra are also consistent with this: we find $\mathrm{Mg}$ $b^{\prime} \propto(0.15 \pm 0.02) \log \sigma$, with a scatter of $0.010 \mathrm{mag}$. [A linear regression of the values of $\mathrm{Mg}_{2}$ and $\mathrm{Mg} b^{\prime}$ in our co-added spectra is well fitted by $\mathrm{Mg}_{2}=(1.70 \pm 0.30) \mathrm{Mg}$ $b^{\prime}-0.01$; this is slightly shallower than the relation found by Colless et al.: $\mathrm{Mg}_{2}=1.94 \mathrm{Mg} b^{\prime}-0.05$.] The $\mathrm{Mg} b^{\prime}-\sigma$ relation in our data evolves: in the highest redshift bins it is about $0.013 \pm 0.002$ mag lower than in the lowest redshift bins. Colless et al. find that in the single stellar population models of both Worthey (1994) and Vazdekis et al. (1996), changes in age or metallicity affect $\mathrm{Mg}_{2}$ about twice as strongly as they do $\mathrm{Mg} b^{\prime}$. Figure 10 suggests that $\mathrm{Mg}_{2}$ has weakened by -0.015 , so we expect $\mathrm{Mg} b^{\prime}$ to have decreased by about -0.007 . Therefore, this also suggests that the $\operatorname{Mg} b$ (or $\mathrm{Mg} b^{\prime}$ ) evolution we see is large.

TABLE 3

Line Index Measurements From Co-Added Spectra

\begin{tabular}{|c|c|c|c|c|c|c|c|c|c|c|c|c|c|}
\hline $\begin{array}{l}\text { ID } \\
\text { (1) }\end{array}$ & $\begin{array}{c}\log \sigma \\
\left(\mathrm{km} \mathrm{s}^{-1}\right) \\
(2)\end{array}$ & $\begin{array}{c}\log R_{o} \\
\left(h_{70}^{-1} \mathrm{kpc}\right) \\
\quad(3)\end{array}$ & $\begin{array}{c}M_{r^{*}} \\
(\mathrm{mag}) \\
(4)\end{array}$ & $\begin{array}{c}z \\
(5)\end{array}$ & $\begin{array}{l}N \text { den } \\
(6)\end{array}$ & $\begin{array}{l}\log H \beta \\
(\AA) \\
(7)\end{array}$ & $\begin{array}{c}\delta \log _{\AA} H \beta \\
(\AA) \\
(8)\end{array}$ & $\begin{array}{c}\mathrm{Mg}_{2} \\
(\mathrm{mag}) \\
(9)\end{array}$ & $\begin{array}{c}\delta \mathrm{Mg}_{2} \\
(\mathrm{mag}) \\
(10)\end{array}$ & $\begin{array}{c}\log \mathrm{Mg} b \\
(\AA) \\
(11)\end{array}$ & $\begin{array}{c}\delta \log \operatorname{Mg} b \\
(\AA) \\
(12)\end{array}$ & $\begin{array}{c}\log \langle\mathrm{Fe}\rangle \\
(\AA) \\
(13)\end{array}$ & $\begin{array}{c}\delta \log _{\circ}\langle\mathrm{Fe}\rangle \\
(\AA) \\
(14)\end{array}$ \\
\hline $2 \ldots \ldots \ldots$ & 2.10 & 0.40 & -20.50 & 0.08 & 2 & 0.219 & 0.005 & 0.264 & 0.001 & 0.594 & 0.002 & 0.490 & 0.002 \\
\hline $3 \ldots \ldots \ldots$ & 2.10 & 0.60 & -21.00 & 0.08 & 2 & 0.256 & 0.005 & 0.251 & 0.002 & 0.609 & 0.003 & 0.515 & 0.005 \\
\hline $4 \ldots \ldots \ldots$ & 2.10 & 0.60 & -21.50 & 0.08 & 2 & 0.242 & 0.005 & 0.260 & 0.002 & 0.586 & 0.003 & 0.489 & 0.002 \\
\hline
\end{tabular}

NotEs.-Table 3 is presented in its entirety in the electronic edition of the Astronomical Journal. A portion is shown here for guidance regarding its form and content. 


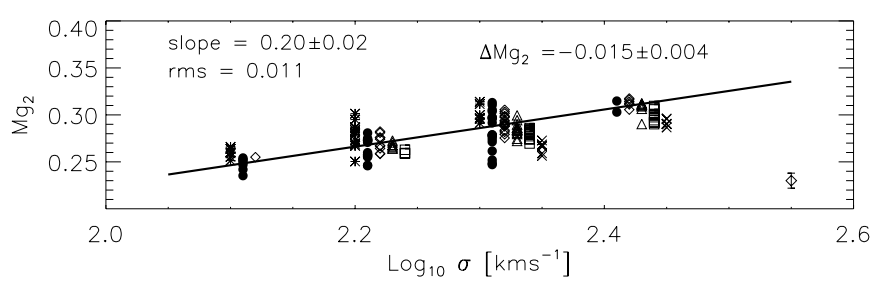

FIg. 10.- $\mathrm{Mg}_{2}$ as a function of $\sigma$. Stars, filled circles, diamonds, triangles, squares, and crosses show results from co-added spectra of similar galaxies in successively higher redshift bins $(z<0.075,0.075<z \leq 0.1,0.1<z \leq$ $0.12,0.12<z \leq 0.14,0.14<z \leq 0.18$, and $z>0.18$ ). Symbol with bar in bottom corner shows the typical uncertainty on the measurements. At fixed redshift, $\mathrm{Mg}_{2}$ increases with increasing $\sigma$. At fixed $\sigma$, the spectra from higher redshift galaxies are weaker in $\mathrm{Mg}_{2}$. Text at top right shows the shift between the lowest and highest redshift bins averaged over the mean shifts at $\log \sigma=2.2,2.3$, and 2.4. We also performed linear fits to the relations at each redshift, and then averaged the slopes, zero points, and rms scatter around the fit. Solid line shows the mean relation obtained in this way, and text at top shows the averaged slope and averaged scatter.

The second panel in Figure 11 shows that, at fixed redshift, $\mathrm{H} \beta \propto \sigma^{-0.24 \pm 0.03}$ with a scatter of 0.027 . This is consistent with Jørgensen (1997), who found $\log \mathrm{H} \beta=$ $(-0.231 \pm 0.082) \log \sigma+0.825$, although our scatter is smaller then her value of 0.061 . At fixed $\sigma, \mathrm{H} \beta$ is stronger in the higher redshift spectra. On average, the value of $\mathrm{H} \beta$ increases by about $0.058 \pm 0.011$ between our lowest and highest redshift bins. An increase of star formation activity with redshift is consistent with a passively evolving population. When a larger sample is available, it will be interesting to see if the scatter in $\mathrm{H} \beta$ at fixed $\sigma$ also increases with redshift.

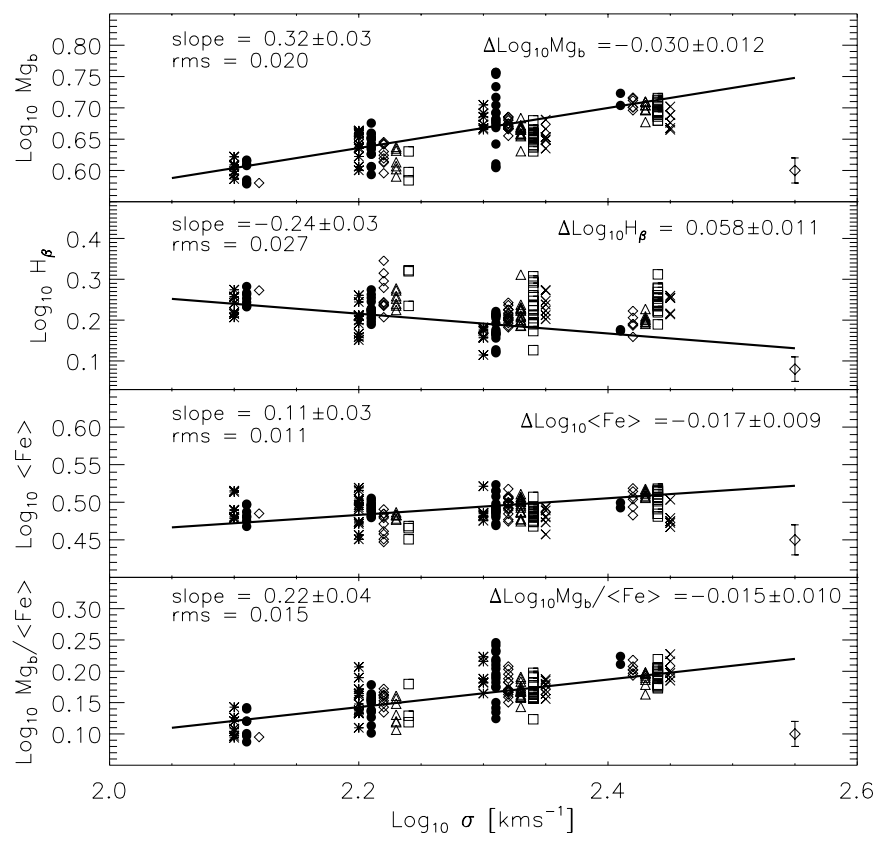

FIG. 11.- Same as Fig. 10, but now showing the spectral line indexes $\mathrm{Mg} b, \mathrm{H} \beta,\langle\mathrm{Fe}\rangle$, and the ratio $[\mathrm{Mg} b / \mathrm{Fe}]$ (top to bottom) as functions of $\sigma$. At fixed redshift, $\mathrm{Mg} b$ and $\langle\mathrm{Fe}\rangle$ increase, whereas $\mathrm{H} \beta$ decreases with increasing $\sigma$. At fixed $\sigma$, the spectra from higher redshift galaxies are weaker in both $\mathrm{Mg}_{2}$ and $\langle\mathrm{Fe}\rangle$, but stronger in $\mathrm{H} \beta$. The text at top right shows the shift between the lowest and highest redshfit bins averaged over the values at $\log \sigma=2.2,2.3$, and 2.4 .
The third panel of Figure 11 shows that, at fixed redshift, $\langle\mathrm{Fe}\rangle \propto \sigma^{0.11 \pm 0.03}$ with a scatter of 0.011 . This lies between the $0.075 \pm 0.025$ scaling and scatter of 0.041 found by Jørgensen (1997), and that found by Kuntschner (2000): $\langle\mathrm{Fe}\rangle \propto \sigma^{0.209 \pm 0.047}$. At fixed $\sigma,\langle\mathrm{Fe}\rangle$ is slightly smaller at higher redshift: the change in $\log \langle\mathrm{Fe}\rangle$ is $0.017 \pm 0.009$.

The ratio $\mathrm{Mg} b /\langle\mathrm{Fe}\rangle$ is sometimes used to constrain models of how early-type galaxies formed (e.g., Worthey, Faber, \& Gonzalez 1992; Thomas, Greggio, \& Bender 1999; but see Matteucci, Ponzone, \& Gibson 1998). In our co-added spectra, $\log \mathrm{Mg} b /\langle\mathrm{Fe}\rangle=(0.22 \pm 0.04) \log \sigma-0.34$ with a scatter of 0.015 (Fig. 11, bottom). The slope of this relation equals the difference between the slopes of the $\mathrm{Mg} b-\sigma$ and $\langle\mathrm{Fe}\rangle-\sigma$ relations, and there is marginal evidence of evolution: the change in $\mathrm{Mg} b /\langle\mathrm{Fe}\rangle$ is $0.015 \pm 0.010$. This correlation should be interpreted as evidence that the contribution of $\mathrm{Fe}$ to the total metallicity is depressed, rather than that the $\alpha$-elements are enhanced, at high $\sigma$ (e.g., Worthey et al. 1992; Weiss, Peletier, \& Matteucci 1995; Greggio 1997; Trager et al. 2000a).

If the evolution in $\mathrm{Mg}$ and $\mathrm{Fe}$ is due to the same physical process, then one might have wondered if residuals from the $\mathrm{Mg} b-\sigma$ relation are correlated with residuals from the $\langle\mathrm{Fe}\rangle-$ $\sigma$ relation. This will be easier to address when the sample is larger. At the present time, we see no compelling evidence for such a correlation - we have not included a figure showing this explicitly. In addition, at any given redshift, galaxies that are richer in $\mathrm{Mg}_{2}$ or $\langle\mathrm{Fe}\rangle$ than they should be (given their velocity dispersion) are neither more nor less likely to be richer in $\mathrm{H} \beta$ than expected - recent star formation is not correlated with metallicity.

\subsection{Line Indices and Color}

Because both the colors and the line indices are evolving, it is interesting to see if the evolution in color and in the indices is similar. The line indices and color both correlate with $\sigma$, and we know how much the individual relations evolve, so we can estimate the evolution in the index-color relation as follows.

Let $y_{0}=s x_{0}+c_{0}$ denote the mean relation between line index $y$ and color $x$ at $z=0$. Because we know that line indices and color both correlate with $\sigma$, and we know how much the individual relations evolve, we can estimate the evolution in the index-color relation by setting $y(z)=y_{0}+$ $\Delta y=s x_{0}+c_{0}+\Delta y=s x(z)+c_{0}+\Delta y-s \Delta x$. For $x=$ $\left(g^{*}-r^{*}\right)$ and $y=\mathrm{Mg}_{2}$, we must set $\Delta y=-0.015, \Delta x=$ -0.042 , and $s=0.20 / 0.26$ (from Table 1 and Fig. 10). Thus, the slope of $\mathrm{Mg}_{2}$ versus $g^{*}-r^{*}$ color should have a slope of 0.77 , and the zero point is expected to evolve by 0.017 between the lowest and highest redshift bins in our sample. A similar analysis for $\mathrm{H} \beta$ and $g^{*}-r^{*}$ suggests that slope is expected to be -0.92 , and the zero point should evolve by 0.019 , whereas the slope of the $\log \langle\mathrm{Fe}\rangle$-color relation should be 0.42 with essentially no evolution. (These estimates assume that the slopes of the individual relations do not evolve. Bender et al. 1996 present some evidence that $\operatorname{Mg} b$ at high $\sigma$ evolves less than at low $\sigma$, suggesting that the slope of the $\mathrm{Mg} b-\sigma$ relation was steeper in the past. A comparison of the $\log \sigma=2.3$ and 2.4 bins in Figure 11 is in approximate agreement with this. Because these estimates are of the order of the error in the measurements, we have not worried about the effects of a change in slope-but with a larger sample, this will be important.) 


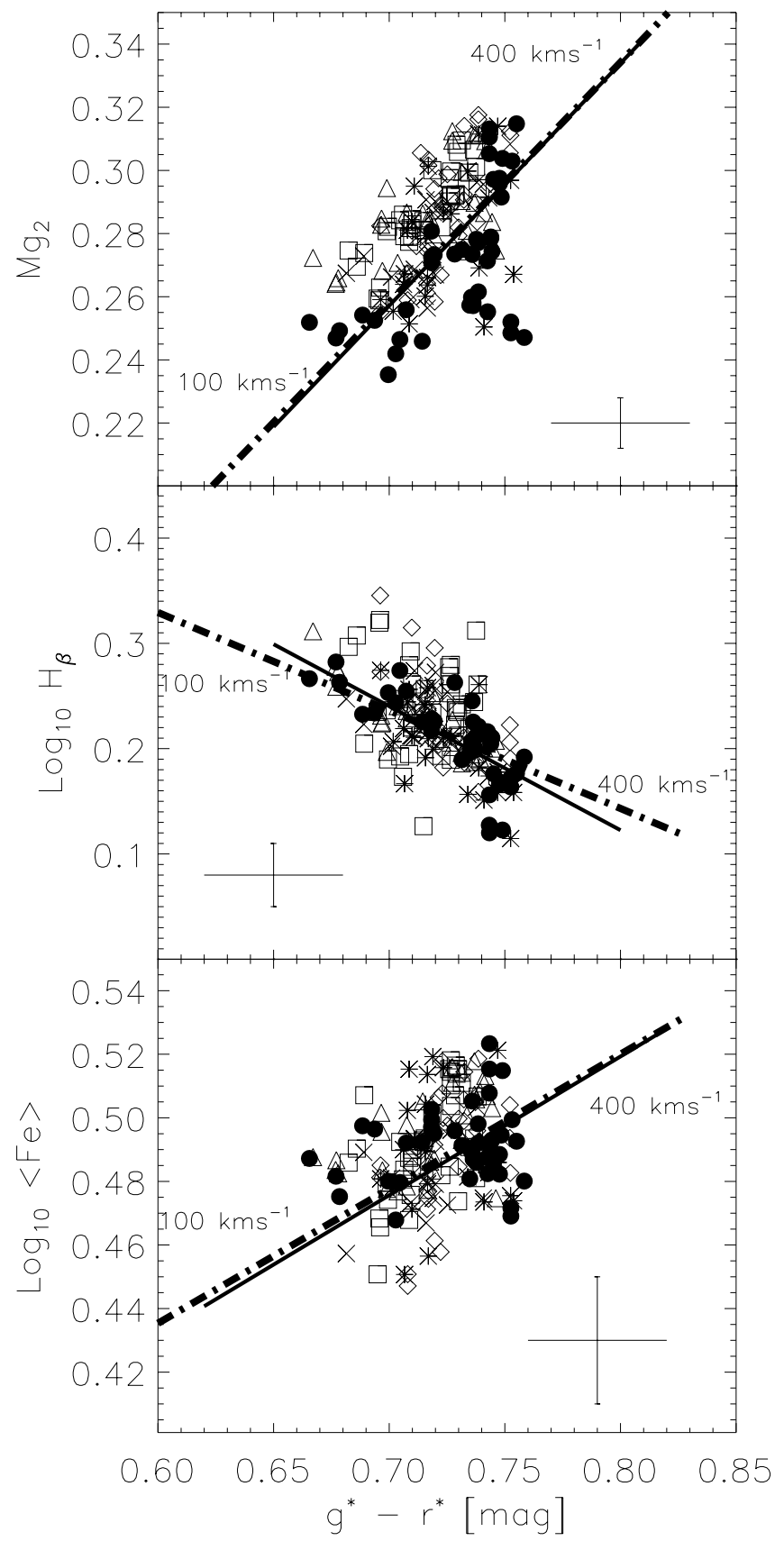

FIG. 12.-Line indices vs. color. Dot-dashed lines show the slope one expects if there were no scatter around the mean color- $\sigma$ and line index $-\sigma$ relations, and solid lines show the linear relation that provides the best fit to the points. Text along the each dot-dashed line indicates the typical value of the velocity dispersion at that location in line index-color space. Crosses in the bottom of each panel show the typical uncertainties. The error in the color is supposed to represent the actual uncertainty in the color, rather than how well the mean color in each bin has been measured.

To check the accuracy of these estimates, Figure 12 shows plots of the line indices versus $g^{*}-r^{*}$ color. (Recall that the line indices were computed from co-added spectra of galaxies that had the same $R_{o}, \sigma$, and $r^{*}$ luminosity. The color here is the mean color of the galaxies in each of those bins.) The solid lines show best fits to the points contributed by the median redshift bins (triangles and diamonds). The dot-dashed lines in each panel show the slopes estimated above; they are not far off from the best-fit lines. (The text in each panel indicates the typical velocity dispersion associated with different locations in index-color space.) The estimates of the evolution of the zero point also appear to be reasonably accurate. The higher redshift crosses in the $\mathrm{Mg}_{2}$ panel appear to lie about 0.02 mag above the lower redshift stars; the difference between the low- and high-redshift populations is obvious. In contrast, the evolution in $\mathrm{H} \beta$ and color conspire so that there is little net offset between the low- and high-redshift populations (note that an offset of $0.02 \mathrm{mag}$ in $\mathrm{Mg}_{2}$ is much more obvious than an offset of 0.02 in $\mathrm{H} \beta$ ). This suggests that the evolution in color and in $\mathrm{H} \beta$ are driven by the same process. Finally, there is little or no offset between the low- and high-redshift bins in $\langle\mathrm{Fe}\rangle$ and $g^{*}-r^{*}$ (bottom), as expected. We have also checked if the residuals from the index-color relations shown in Figure 12 correlate with local density: they do not.

\subsection{Comparison with Stellar Population Models}

The different line index- $\sigma$ and color- $\sigma$ relations are evolving. Stellar population models can be used to study what the evolution we see implies.

The predictions of single age stellar population models (e.g., Bruzual \& Charlot 1993; Worthey 1994; Vazdekis et al. 1996; Tantalo, Chiosi, \& Bressan 1998) are often summarized as plots of $\mathrm{H} \beta$ versus $\mathrm{Mg} b$ (or $\mathrm{Mg}_{2}$ ) and $\langle\mathrm{Fe}\rangle$. The usual caveats noted by these authors about the limitations of these models, and the assumption that all the stars formed in a single burst, apply. In addition, comparison with data is complicated because the models assume that the ratio of $\alpha$-elements to Fe peak elements in early-type galaxies is the same as in the Sun, whereas, in fact, it differs from the solar value by an amount that depends on velocity dispersion (e.g., Worthey et al. 1992; see Fig. 11, bottom). We use a simplified version of the method described by Trager et al. (2000a) to account for this.

Figure 13 shows such a plot. The dotted grids (top and bottom left are the same, as are top and bottom right) show a single-age, solar abundance (i.e., $[\mathrm{E} / \mathrm{Fe}]=0$ ), stellar population model (from Worthey 1994); lines of constant age run approximately horizontally (top to bottom show ages of 2, 5, 8, 12, and $17 \mathrm{Gyr}$ ), lines of constant metallicity run approximately vertically (left to right show $[\mathrm{Fe} / \mathrm{H}]=$ $-0.25,0,0.25,0.5)$. Points in the panels on the top show the values of $\mathrm{H} \beta$ and $\mathrm{Mg} b$ (left) and $\langle\mathrm{Fe}\rangle$ (right) for the coadded spectra in our sample. Different symbols show different redshift bins; the higher redshift population (squares and crosses) appears to show a larger range in $\mathrm{H} \beta$ compared with the low-redshift population (stars and circles). The cross in each panel shows the typical uncertainty on the measurements.

The heavy dot-dashed lines in the top panels show the relation between $\mathrm{H} \beta$ and $\mathrm{Mg} b$ or $\langle\mathrm{Fe}\rangle$ one predicts if there were no scatter around the individual line index- $\sigma$ relations (Fig. 11, solid lines): $\mathrm{H} \beta \propto \mathrm{Mg} b^{-0.75}$ and $\mathrm{H} \beta \propto\langle\mathrm{Fe}\rangle^{-2.2}$. We have included them to help disentangle the evolution we saw in the individual line index- $\sigma$ relations from the effect of the magnitude limit. The text shows the typical velocity dispersion associated with the location in "index-index" space. The evolution in the $\mathrm{Mg} b-\sigma,\langle\mathrm{Fe}\rangle-\sigma$, and $\mathrm{H} \beta-\sigma$ relations suggest that the higher redshift sample should be displaced upward and to the left, with a net shift in zero point of about 0.03 and 0.02 in the upper left and right panels of 


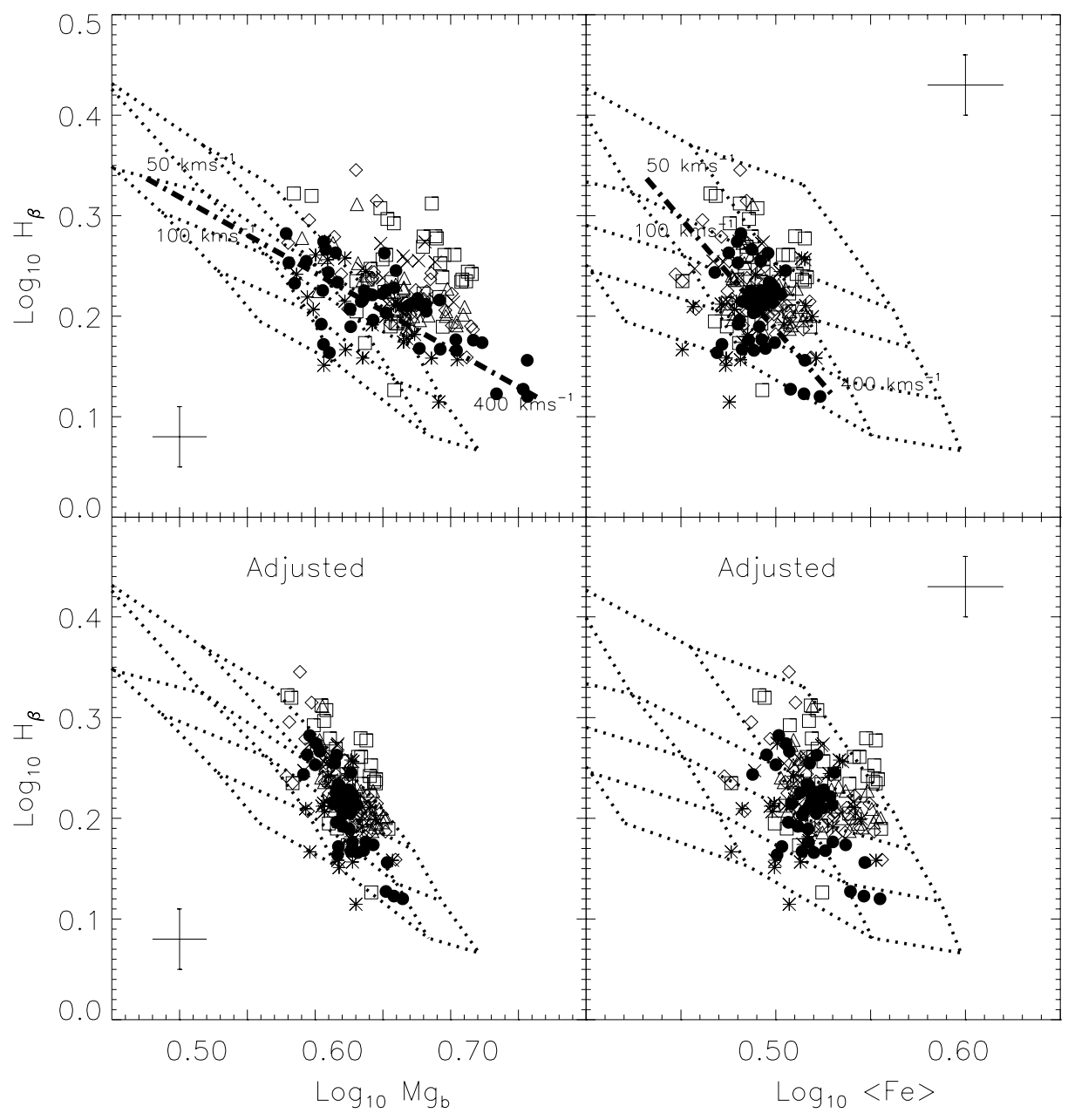

FIG. 13.- $\mathrm{H} \beta$ vs. $\mathrm{Mg} b$ (left) and $\langle\mathrm{Fe}\rangle$ (right) for the co-added spectra in our sample. Different symbols show different redshift bins; the higher redshift population (squares and crosses) appears to show a larger range in $\mathrm{H} \beta$ compared with the low-redshift population (stars and circles). Cross in each panel shows the typical uncertainty on the measurements. Dotted grid shows a single age, solar abundance (i.e., $[\mathrm{E} / \mathrm{Fe}]=0$ ), stellar population model (from Worthey 1994); lines of constant age run approximately horizontally (top to bottom: ages of $2,5,8,12$, and $17 \mathrm{Gyr}$ ), lines of constant metallicity run approximately vertically (left to right: $[\mathrm{Fe} / \mathrm{H}]=-0.25,0,0.25$, and 0.5 ). The two top panels provide different estimates of the age and metallicity, presumably because the $[\mathrm{E} / \mathrm{Fe}]$ abundances in our data are different from solar. In the bottom panels, this difference has been accounted for, and the age and metallicity estimates agree. Dot-dashed line in top panels shows what one expects if there is no scatter around the line index $-\sigma$ relations (Fig. 11, solid lines); text shows the typical velocity dispersion associated with the location in "index-index" space.

Figure 13. (We estimate these shifts similarly to how we estimated the evolution in the line index-color relations.)

Although the expected evolution is slightly smaller than the typical uncertainty in the measurements, the top two panels in Figure 13 do appear to show that the high-redshift population (squares and crosses) is displaced slightly upward. The shift to the left is not apparent, however, because of the selection effect: evolution moves the high- $\sigma$ objects of the high-redshift sample onto the lower $\sigma$ points of the low-redshift sample, but the low- $\sigma$ objects at higher redshift, which would lie clearly above and to the left, are not seen because of the selection effect. Note that the selection effect works so that evolution effects are suppressed, rather than enhanced in plots like Figure 13; therefore, a simple measurement of evolution in the top panels should be interpreted as a lower limit to the true value.

The top two panels show that our sample spans a range of about 0.3 or more in metallicity and a large range of ages. However, note that the two panels provide different estimates of the mean ages and metallicities in our sample. This is because the $[\mathrm{E} / \mathrm{Fe}]$ abundances in our data are different from solar. Trager et al. (2000a) describe how to correct for this. Our measurement errors in $\mathrm{Mg}$ and $\mathrm{Fe}$ are larger than theirs, so we have adopted the following simplified version of their prescription.

Let $[Z(\mathrm{H} \beta,\langle\mathrm{Fe}\rangle) / \mathrm{H}]$ denote the estimate of the metallicity given by the top right panel of Figure 13: this estimate uses the observed values of $\mathrm{H} \beta$ and $\langle\mathrm{Fe}\rangle$ and the Worthey (1994) solar abundance ratio models. Trager et al. (2000a) argue that nonsolar abundances change the relation between $[\mathrm{Fe} / \mathrm{H}]$ and the true metallicity $[Z / \mathrm{H}]:[\mathrm{Fe} / \mathrm{H}]=[Z / \mathrm{H}]-$ $A[\mathrm{E} / \mathrm{Fe}]$, where $A \approx 0.93$. Trager et al. (2000b) argue that $[\mathrm{E} / \mathrm{H}] \approx 0.33 \log \sigma-0.58$, and that the relation is sufficiently tight that one can substitute $\sigma$ for $[\mathrm{E} / \mathrm{Fe}]$. Although we have not measured this relationship between $[\mathrm{E} / \mathrm{Fe}]$ and velocity dispersion in our sample, we assume it is accurate. This allows us to define a corrected metallicity $[Z / H]_{\text {corr }} \approx$ $[Z(\mathrm{H} \beta,\langle\mathrm{Fe}\rangle) / \mathrm{H}]+0.33 A(\log \sigma-0.58)$. Trager et al. (2000a) also argue that correcting for nonsolar $[\mathrm{E} / \mathrm{Fe}]$ makes a negligible change to $\mathrm{H} \beta$. Therefore, we combine the measured value of $\mathrm{H} \beta$ with $[Z / \mathrm{H}]_{\text {corr }}$ to compute a corrected age $\tau_{\text {corr }}$. We then use Worthey's model with these 
corrected ages and metallicities to obtain corrected values of $\mathrm{Mg} b$ and $\langle\mathrm{Fe}\rangle$. These are plotted in the bottom panels. By construction, the values of $\mathrm{H} \beta$ in all four panels are the same, and the age and metallicity estimates in the bottom two panels agree. The differences between our top and bottom panels are similar to the differences between Figures 1 and 3 of Trager et al. (2000a), suggesting that our simple approximate procedure is reasonably accurate.

We apply the same correction procedure to plots of $\mathrm{H} \beta-\mathrm{Mg}_{2}$ and $\langle\mathrm{Fe}\rangle-\mathrm{Mg}_{2}$ in Figure 14. The dot-dashed lines in the panels on the left show $\log \mathrm{H} \beta \propto-1.20 \mathrm{Mg}_{2}$ and $\log \langle\mathrm{Fe}\rangle \propto 0.55 \mathrm{Mg}_{2}$. Matteucci et al. (1998) report that a fit to a compilation of $\langle\mathrm{Fe}\rangle-\mathrm{Mg}_{2}$ data from various sources has slope 0.6. The dot-dashed line does not appear to provide a good fit in the top panel, although this may be because of a combination of evolution and selection effects: fitting the relation separately for different redshift bins and averaging the results yields a line that is more like the dot-dashed line.

The expected evolution is upward and to the left for $\mathrm{H} \beta$ $\mathrm{Mg}_{2}$ and down and left for $\langle\mathrm{Fe}\rangle-\mathrm{Mg}_{2}$, with net shifts in zero points of 0.040 and -0.009 . Thus, in the bottom panel, evolution moves points along the dot-dashed line. As with the previous plot, the selection effect makes evolution difficult to see. The dotted grid shows the Worthey (1994) model for these relations. Comparison of the two bottom panels suggests that much of the scatter in the observed $\langle\mathrm{Fe}\rangle-\mathrm{Mg}_{2}$ relation is due to differences in enhancement ratios.

We can now use the models to estimate the mean corrected ages and metallicities of the galaxies in our sample as a function of redshift. The mean metallicity is 0.33 and shows almost no evolution. The mean age in our lowest redshift bin (stars, median redshift 0.06 ) is $8 \mathrm{Gyr}$, whereas it is 6 Gyr in the highest redshift bin (crosses). The redshift difference corresponds to a time interval of $1.63 \mathrm{Gyr}$; if the population has evolved passively, this should equal the difference in ages from the stellar population models. While the numbers are reasonably close, it is important to note that, because of the magnitude limit, our estimates of the typical age and metallicity at high redshift are biased toward high values, whereas our estimate of the evolution relative to the population at low redshift is probably biased low. Nevertheless, it is reassuring that this estimate of a formation time of 8 or $9 \mathrm{Gyr}$ ago is close to that which we use to make our $K$-corrections.

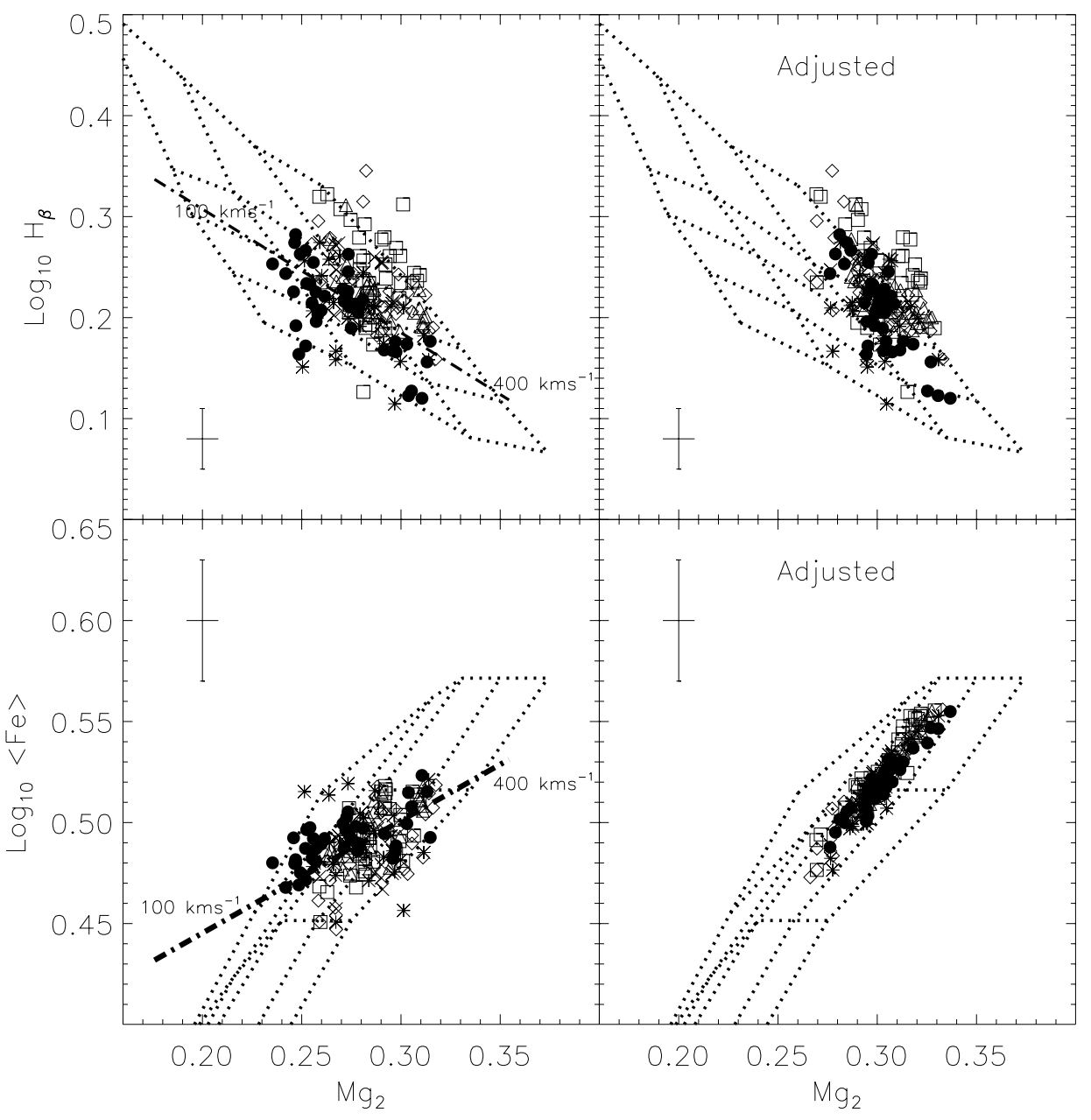

FIg. 14.- Line indices $\mathrm{H} \beta$ and $\langle\mathrm{Fe}\rangle$ vs. $\mathrm{Mg}_{2}$ for the co-added spectra in our sample. Symbols (same as Fig. 13) show results for different redshift bins. Dot-dashed line shows the relation one predicts if there were no scatter around the individual line index $-\sigma$ relations; text shows the typical velocity dispersion associated with the location in "index-index" space. Evolution is expected to move points upward and to the left for $\mathrm{H} \beta$ vs. $\mathrm{Mg}_{2}$ (top), but downward and left, and along the dot-dashed line in the case of $\langle\mathrm{Fe}\rangle$ and $\mathrm{Mg}_{2}$ (bottom), although selection effects make these trends difficult to see. Dotted grids show the same single stellar population model as in the previous figure (from Worthey 1994). Age and metallicity estimates in the top panels are inconsistent with those in the bottom panels if solar abundance is assumed $(l e f t)$, but the estimates agree once differences in abundances have been accounted for $(r i g h t)$. 


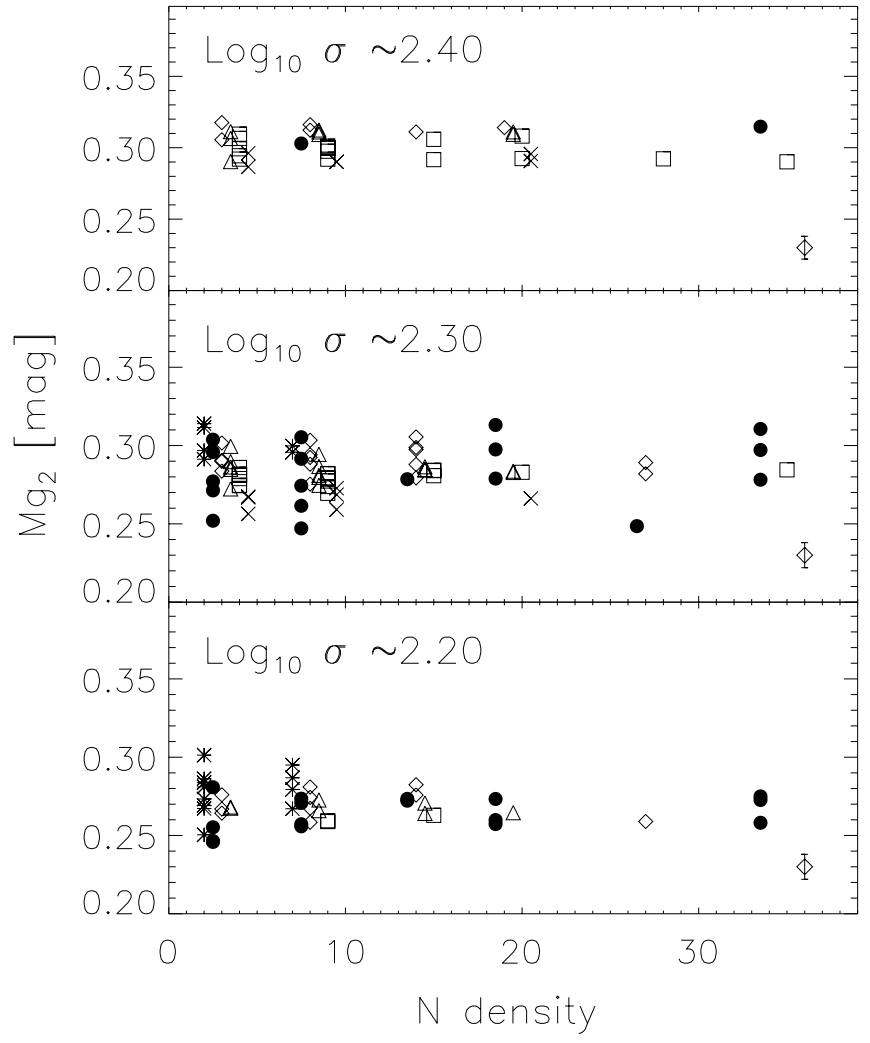

FIG. 15.- $\mathrm{Mg}_{2}$-density relation for the galaxies in our sample. Symbols show the different redshift bins; higher redshift bins have been offset slightly to the right. Symbol with bar in bottom right shows the typical uncertainty on the measurements.

\subsection{Dependence on Environment}

We now turn to a study of how the co-added spectra depend on environment. Figures 15 and 16 show the strength of $\mathrm{Mg}_{2}$ and $\mathrm{H} \beta$ in a few small bins in $\sigma$, as a function of local density. The different symbols in each density bin represent composite spectra from different redshiftshigher redshift bins have offset slightly to the right. This allows us to separate the effects of evolution from those of environment. Figure 15 shows that, at fixed $\sigma, \mathrm{Mg}_{2}$ decreases with redshift. At any given redshift, the strength of $\mathrm{Mg}_{2}$ is independent of local density. (Our sample is not large enough to say with certainty if the evolution depends on environment.) A similar plot for $\mathrm{H} \beta$ also shows strong evolution with redshift and no dependence on environment (Fig. 16). Similar plots of $\langle\mathrm{Fe}\rangle$ and $\left[\mathrm{Mg}_{2} / \mathrm{Fe}\right]$ also show little, if any, dependence on redshift and no dependence on environment, so we have not shown them here.

We caution that our definition of environment is limited, because it is defined by early-type galaxies only. In addition, because we must divide our total sample up into bins in luminosity, size, radius, and redshift, and then by environment, the statistical significance of the results here would be greatly improved by increasing the sample size. Analysis of environmental dependence using a larger sample is presented in Eisenstein et al. (2003).

In conclusion, although we have evidence from the fundamental plane that early-type galaxies in dense regions are slightly different from their counterparts in less dense regions (Fig. 9 in Paper III; also see Fig. 10 in Paper I), these differences are sufficiently small that the strengths of spec-

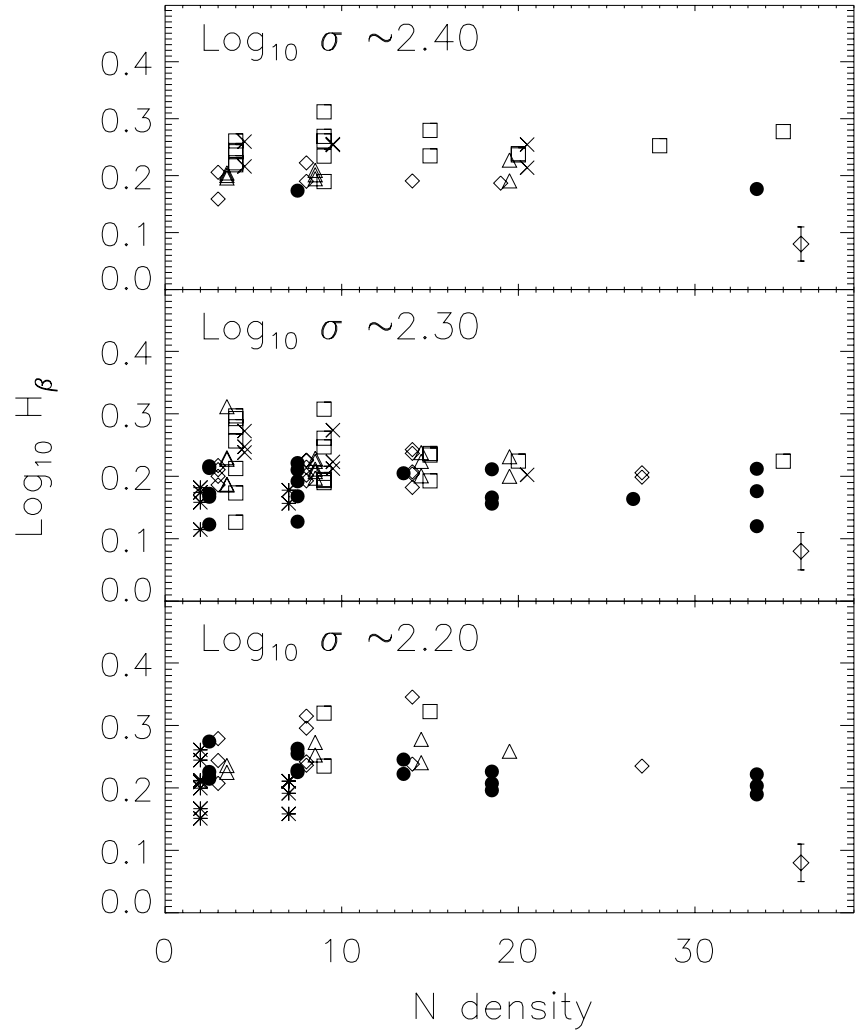

Fig. 16. - Same as for Fig. 15, but for the $\mathrm{H} \beta$-density relation. At fixed velocity dispersion, $\mathrm{H} \beta$ is slightly higher at high redshift, but there is no significant dependence on environment.

tral features are hardly affected (Figs. 15 and 16). However, the co-added spectra provide strong evidence that the chemical composition of the population at low and high redshifts is different (Figs. 10-14).

\section{DISCUSSION AND CONCLUSIONS}

We have studied $\sim 9000$ early-type galaxies over the redshift range $0 \leq z \leq 0.3$ using photometric (in the $g^{*}, r^{*}, i^{*}$, and $z^{*}$ bands) and spectroscopic observations. The colors of the galaxies in our sample are strongly correlated with velocity dispersion - redder galaxies have larger velocity dispersions ( $(2)$. The color-magnitude and color-size relations are a consequence of the fact that $M$ and $R_{o}$ also correlate with $\sigma$ (Fig. 3). The strength of the color-magnitude relation depends strongly on whether or not fixed apertures were used to define the colors, whereas the color- $\sigma$ relation appears to be less sensitive to these differences (Figs. 7 and 8). At fixed velocity dispersion, the population at high redshift is bluer than that nearby (Figs. 1 and 2), and the evolution in color is significantly less than that of the luminosities (Table 1). A larger sample, with well-understood $K$ corrections, is required to quantify if galaxies in denser regions are slightly redder and more homogeneous or not (Figs. 4 and 5).

The SDSS spectra of individual galaxies do not have extremely high values of signal-to-noise ratio (typically $\mathrm{S} / \mathrm{N} \sim 15$; cf. Fig. 18 in Paper I). However, the data set is so large that we were able to study stellar population indicators $\left(\mathrm{Mg}_{2}, \mathrm{Mg} b,\langle\mathrm{Fe}\rangle\right.$, and $\left.\mathrm{H} \beta\right)$ by co-adding the spectra of early-type galaxies that have similar luminosities, sizes, 
velocity dispersions, environments, and redshifts to create a composite with higher $\mathrm{S} / \mathrm{N}$ spectra. The resulting library of 182 composite spectra, all of which have $\mathrm{S} / \mathrm{N}>50$, and many of which have $\mathrm{S} / \mathrm{N}>100$, covers a large range of velocity dispersions, sizes, and luminosities. It is available electronically.

All the line indices correlate with velocity dispersion $(\S 3)$ : $\mathrm{Mg}_{2} \propto \sigma^{0.20}, \mathrm{Mg} b \propto \sigma^{0.32},\langle\mathrm{Fe}\rangle \propto \sigma^{0.11}$, and $\mathrm{H} \beta \propto \sigma^{-0.24}$. These correlations are consistent with those in the literature, although the results from the literature were obtained from individual, as opposed to co-added, galaxy spectra. The co-added spectra show no significant dependence on environment. However, the spectra show clearly that, at fixed velocity dispersion, the high-redshift population is stronger in $\mathrm{H} \beta$ and weaker in $\mathrm{Mg}$ and $\mathrm{Fe}$ than the population at lower redshifts (Figs. 10-14). Line indices also correlate with color: a good approximation to these correlations is obtained by using the fact that line indices and color both correlate strongly with velocity dispersion and ignoring the scatter.

Single burst stellar population models (e.g., Worthey 1994; Vazdekis et al. 1996) allow one to translate the evolution in the spectral features into estimates of the ages and metallicities of the galaxies in our sample (e.g., Trager et al. $2000 \mathrm{a}, 2000 \mathrm{~b}$ ). In our sample, the $z \approx 0.05$ population appears to about $8 \mathrm{Gyr}$ old; the $z \approx 0.2$ population in our sample appears to be about 2 Gyr younger; and the average metallicity appears to be similar in both populations. The age difference is approximately consistent with the actual time difference in the $\left(\Omega_{M}, \Omega_{\Lambda}, h\right)=(0.3,0.7,0.7)$ world model we assumed throughout this paper, suggesting that the population is evolving passively. Given a formation time, the single burst stellar population models also make predictions about how the luminosities and colors should evolve with redshift. Our estimates of this evolution are also consistent with those of a population that formed the bulk of its stars 9 Gyr ago.
By the time SDSS is complete, the uncertainty in the $K$ corrections, which prevent us at the present time from making more precise quantitative statements about the evolution of the luminosities and colors, will be better understood. In addition, the size of the sample will have increased by more than an order of magnitude. This will allow us to provide a more quantitative study of the effects of environment than we are able to at the present time. Most importantly, a larger sample will allow us to co-add spectra in finer bins; this will allow us to make maximum likelihood estimates, rather than simple linear regression studies, of how features in the spectra correlate with other observables. This should also allow us to address the important issue of whether or not the most luminous galaxies in the population are evolving similarly to the faintest.

We would like to thank S. Charlot for making his stellar population synthesis predictions for the SDSS filters available to the collaboration. Funding for the creation and distribution of the SDSS Archive has been provided by the Alfred P. Sloan Foundation, the Participating Institutions, the National Aeronautics and Space Administration, the National Science Foundation, the US Department of Energy, the Japanese Monbukagakusho, and the Max Planck Society. The SDSS Web site is http:// www.sdss.org/. The SDSS is managed by the Astrophysical Research Consortium (ARC) for the Participating Institutions. The Participating Institutions are the University of Chicago, Fermilab, the Institute for Advanced Study, the Japan Participation Group, Johns Hopkins University, Los Alamos National Laboratory, the Max-Planck-Institute for Astronomy, the Max-Planck-Institute for Astrophysics, New Mexico State University, University of Pittsburgh, Princeton University, the United States Naval Observatory, and the University of Washington.
Baum, W. A. 1959, PASP, 71, 106

Bender, R., Ziegler, B., \& Bruzual A., G. 1996, ApJ, 463, L51

Bernardi, M., Alonso, M. V., da Costa, L. N., Willmer, C. N. A., Wegner, G., Pellegrini, P. S., Rité, C., \& Maia, M. A. G. 2002, AJ, 123, 2990

Bernardi, M., Renzini, A., da Costa, L. N., Wegner, G., Alonso, M. V.,

Pellegrini, P. S., Rite, C., \& Willmer, C. N. A. 1998, ApJ, 508, L143

Bernardi, M., et al. 2003a, AJ, 125, 1817

. 2003b, AJ, 125, 1849 2003c, AJ, 125, 1866

Blakeslee, J. P., Lucey, J. R., Barris, B. J., Hudson, M. J., \& Tonry, J. L.

2001, MNRAS, 327, 1004

Bower, R. G., Lucey, J. R., \& Ellis, R. S. 1992a, MNRAS, 254, 589 1992b, MNRAS, 254, 601

Bruzual A., G., \& Charlot, S. 1993, ApJ, 405, 538

Coleman, G. D., Wu, C.-C., \& Weedman, D. W. 1980, ApJS, 43, 393

Colless, M., Burstein, D., Davies, R. L., McMahan, R. K., Saglia, R. P., \& Wegner, G. 1999, MNRAS, 303, 813

de Vaucouleurs, G. 1961, ApJS, 5, 233

Eisenstein, D. F., et al. 2003, ApJ, in press

Fioc, M., \& Rocca-Volmerange, B. 1999, A\&A, 351, 869

Greggio, L. 1997, MNRAS, 285, 151

Jørgensen, I. 1997, MNRAS, 288, 161

Kodama, T., Bower, R. G., \& Bell, E. F. 1999, MNRAS, 306, 561

\section{REFERENCES}

Kuntschner, H. 2000, MNRAS, 315, 184

Matteucci, F., Ponzone, R., \& Gibson, B. K. 1998, A\&A, 335, 855

Okamura, S., et al. 1998, in IAU Symp. 179, New Horizons from Multi-

Wavelength Sky Surveys, ed B. J. McLean, D. A. Golombek, J. J. E.

Hayes, \& H. E. Payne (Dordrecht: Kluwer), 287

Pahre, M. A., et al. 1998, AJ, 116, 1606

Sandage, A., \& Visvanathan, N. 1978a, ApJ, 223, 707

. 1978b, ApJ, 225, 742

Scodeggio, M. 2001, AJ, 121, 2413

Stoughton, C., et al. 2002, AJ, 123, 485

Tantalo, R., Chiosi, C., \& Bressan, A. 1998, A\&A, 333, 419

Thomas, D., Greggio, L., \& Bender, R. 1999, MNRAS, 302, 537

Trager, S. C., Faber, S. M., Worthey, G., \& González, J. J. 2000a, AJ, 119, 1645

2000b, AJ, 120, 165

Trager, S. C., Worthey, G., Faber, S. M., Burstein, D., \& González, J. J. 1998, ApJS, 116, 1

Vazdekis, A., Casuso, E., Peletier, R. F., \& Beckman, J. E. 1996, ApJS, 106,307

Weiss, A., Peletier, R. F., \& Matteucci, F. 1995, A\&A, 296, 73

Worthey, G. 1994, ApJS, 95, 107

Worthey, G., Faber, S. M., \& Gonzales, J. 1992, ApJ, 398, 69 